\title{
Wigner function and kinetic phenomena for chiral plasma in a strong magnetic field
}

\author{
E.V. Gorbar, ${ }^{a, b}$ V.A. Miransky, ${ }^{c}$ I.A. Shovkovy ${ }^{d, e, 1}$ and P.O. Sukhachov ${ }^{c}$ \\ ${ }^{a}$ Department of Physics, Taras Shevchenko National Kiev University, \\ Kiev, 03680, Ukraine \\ ${ }^{b}$ Bogolyubov Institute for Theoretical Physics, \\ Kiev, 03680, Ukraine \\ ${ }^{c}$ Department of Applied Mathematics, Western University, \\ London, Ontario N6A 5B\%, Canada \\ ${ }^{d}$ College of Integrative Sciences and Arts, Arizona State University, \\ Mesa, Arizona 85212, U.S.A. \\ e Department of Physics, Arizona State University, \\ Tempe, Arizona 85287, U.S.A. \\ E-mail: gorbar@bitp.kiev.ua, vmiransk@uwo.ca, igor.shovkovy@asu.edu, \\ psukhach@uwo.ca
}

ABSTRACT: By using the exact solutions of the Weyl equation in a constant magnetic field, the equal-time Wigner function for magnetized chiral plasma is derived. It is found that the dependence of the Wigner function on the component of momentum along the magnetic field is asymmetric and is correlated with the fermion chirality. Such a dependence is principal for reproducing the correct chiral magnetic and chiral separation effects. In the lowest Landau level approximation, the equation for the equal-time Wigner function in a strong magnetic field is derived. By making use of this equation, it is found that the longitudinal collective modes in a strong magnetic field are gapped plasmons whose gap is determined by the magnetic field. Unlike the ordinary magnetic field, an axial one allows for the dispersion law of the collective excitations asymmetric in the wave vector. The thermoelectric phenomena for chiral fermions in strong magnetic and axial magnetic fields are studied and the corresponding transport coefficients are calculated.

Keywords: Anomalies in Field and String Theories, Quark-Gluon Plasma, Thermal Field Theory, Topological States of Matter

ArXiv EPrint: 1707.01105

\footnotetext{
${ }^{1}$ Corresponding author.
} 


\section{Contents}

1 Introduction 1

2 Model 3

3 Wigner function in a constant magnetic field 4

$\begin{array}{lll}3.1 & \text { Chiral magnetic and chiral separation effects } & 8\end{array}$

$\begin{array}{llr}3.2 & \text { Weak magnetic field expansion } & 9\end{array}$

4 Equation of motion for the Wigner function 10

5 The chiral magnetic and pseudomagnetic waves in a strong magnetic field $\quad 12$

6 Thermoelectric phenomena in a strong magnetic field 14

$\begin{array}{lll}7 & \text { Summary } & 17\end{array}$

A Wave functions of the Weyl Hamiltonian 18

B Derivation of the Wigner function in a constant magnetic field 20

C Weak magnetic field limit $\quad 23$

D Useful formulas $\quad 25$

\section{Introduction}

Kinetic theory $[1,2]$ is the standard and surprisingly very efficient method of the investigation of transport phenomena in various physical systems. Classical kinetic theory is widely used to describe the fluid dynamics, electromagnetic collective excitations in plasmas, conductivity in metals, etc. Quantum kinetic theory is indispensable in the study of transport phenomena in material media at low temperature. Relativistic kinetic theory is relevant in the studies of the primordial plasma of the early Universe [3, 4], relativistic heavy-ion collisions [5, 6], compact stars [7], and the recently discovered Dirac [8-14] and Weyl [15-22] materials whose low-energy excitations are described by the relativistic-like Dirac and Weyl equations, respectively.

One of the qualitatively new key ingredients in the chiral plasma is the chiral charge, whose conservation is violated only by the chiral quantum anomaly [23, 24]. Recently, it was shown that its dynamical evolution can be described using the framework of the kinetic theory. The corresponding version of the theory, i.e., the chiral kinetic theory was formulated in refs. [25-27]. This theory relies on the wave-packet semiclassical description of the anomalous Hall effect [28] in metals which takes into account the Berry curvature effects $[29,30]$. The latter are relevant for the chiral kinetic theory because the Weyl nodes act as the sources and sinks of the Berry curvature. The chiral kinetic theory was 
derived in the first order in the Planck constant $\hbar$ and its equations are linear in electric as well as magnetic fields. Such a theory successfully incorporates the chiral anomaly [23, 24] and describes the chiral magnetic effect [31]. However, many physical phenomena require the chiral kinetic theory accurate to higher orders in the electromagnetic field. The first step in this direction was done in refs. [32, 33] where, by using the wave-packet semiclassical approach, the chiral kinetic equation valid to the second order in a magnetic field was derived for fermions with a general band structure. In our recent paper [34], we provided the explicit expressions for the chiral kinetic theory accurate to the second order in electromagnetic and axial or pseudoelectromagnetic fields for a simple realization of relativistic matter in a Weyl material with a single pair of Weyl nodes.

Certainly, it would be very useful to formulate the equations of the chiral kinetic theory to all orders in electromagnetic fields. A convenient starting point for their derivation is the equation of motion for the Wigner function [35] (see also refs. [36-40]) in an electromagnetic field. In a many-body system, the Wigner function is given by the Fourier transform of the two-point lesser Green's function with respect to the relative coordinates (see, e.g., ref. [41]). Therefore, similarly to the usual distribution function, the Wigner function describes the dynamics in the phase space, albeit retaining all its quantum aspects.

The equation of motion for the Wigner function in an electromagnetic field is exact and mathematically equivalent to the Dirac or Weyl equation. By solving this equation in the perturbation theory in electromagnetic field with the zero-order Wigner function proportional to a combination of the Fermi-Dirac distribution functions, the standard equations of the chiral kinetic theory were derived in ref. [42]. On the other hand, it is very well known that the Dirac or Weyl equation is exactly solvable in a constant magnetic field. This suggests a possible means to analyse the kinetic phenomena for chiral fermions when the background magnetic field is taken into account nonperturbatively and the corresponding Wigner function is found exactly. Further, this solution can be used to analyze perturbatively the effects of a weak electric field, as well as inhomogeneous and time-dependent magnetic fields. This provides the main motivation for the study performed in this paper. In addition, we would like to mention also that such an approach could, in principle, be extended to the case of a constant background electric field. However, unlike the magnetic field, the electric field does work. Therefore, even a stationary solution would describe a nonequilibrium state, in which the pair production can take place. Using the formalism of the equal-time Wigner function (known also as the Dirac-Heisenberg-Wigner function), this problem was analyzed in refs. $[43,44]$. The rearrangement of the particle occupation numbers makes the analysis more complicated, therefore, in this paper we consider only the case of constant background magnetic and axial magnetic fields.

By making use of the equal-time Wigner function, in this study we also address the thermoelectric properties of chiral plasma in the strong magnetic field limit. In weak fields, the corresponding anomalous transport was studied in refs. $[45,46]$ by taking into account the Berry phase effects. Later, these thermoelectric properties were investigated in the framework of the chiral kinetic theory for Dirac and Weyl materials in refs. [47-51]. The authors of ref. [47] showed that the thermoconductivity has the standard linear temperature dependence expected for a metallic system. In the case where the temperature gradient 
and weak magnetic field are parallel, the longitudinal thermoconductivity is quadratic in the magnetic field strength, which is somewhat similar to the quadratic correction to the electric conductivity from the chiral anomaly. If the magnetic field is perpendicular to the temperature gradient, then the magnetic field correction to the thermoconductivity is also quadratic, albeit has a negative sign. In this study, we extend the analysis of the thermoelectric properties of chiral matter to the case of strong background magnetic and axial magnetic fields.

The paper is organized as follows. The model is described in section 2. The equaltime Wigner function for chiral fermions in background constant magnetic and axial (or pseudo-) magnetic fields is explicitly calculated in section 3 . In the same section we tested the obtained Wigner function by studying the chiral magnetic and chiral separation effects, as well as determining its weak magnetic field expansion. The equations of motion for the equal-time Wigner function in the lowest Landau level approximation are given in section 4. The collective excitations as well as the charge and heat transport in the longitudinal direction with respect to the magnetic field are analyzed in sections 5 and 6 , respectively. The results are summarized in section 7 . Some technical details of the derivation and analysis are collected in several appendices at the end of the paper.

Throughout this paper, we set $\hbar=1$ and $c=1$.

\section{Model}

The Weyl Hamiltonian for the right-handed $\lambda=+$ and left-handed $\lambda=-$ fermions in a constant magnetic field is given by

$$
H_{\lambda}=-i \lambda v_{F}(\boldsymbol{\sigma} \cdot \boldsymbol{\nabla})+\lambda v_{F} e\left(\boldsymbol{\sigma} \cdot \mathbf{A}_{\lambda}\right)+\mu_{\lambda}
$$

where $v_{F}$ equals the velocity of light $c$ in the case of relativistic matter or the Fermi velocity of quasiparticles in a Weyl semimetal. Further, $\boldsymbol{\sigma}=\left(\sigma_{x}, \sigma_{y}, \sigma_{z}\right)$ are the Pauli matrices, $\mathbf{A}_{\lambda}=\left(0, x_{1} B_{\lambda}, 0\right)$ is a vector potential for an effective magnetic field $\mathbf{B}_{\lambda}=\mathbf{B}+\lambda \mathbf{B}_{5}$ that points in the $+z$ direction, and $\mu_{\lambda}=\mu+\lambda \mu_{5}$ is an effective chemical potential, where $\mu$ is the fermion number chemical potential and $\mu_{5}$ is the chiral chemical potential. Here, we included the interaction of the chiral fermions with an axial magnetic (or, equivalently, pseudomagnetic) field $\mathbf{B}_{5}$. Such a field interacts with the different sign depending on the fermion chirality. While this field is typically absent in relativistic matter systems (except for, possibly, in the primordial plasma of the early Universe before the electroweak transition) it can be easily induced by mechanical strains in Weyl and Dirac semimetals [5258]. The characteristic strengths of the pseudomagnetic field in Dirac and Weyl materials range from about $B_{5} \approx 0.3 \mathrm{~T}$, when a static torsion is applied to a nanowire of $\mathrm{Cd}_{3} \mathrm{As}_{2}$ [57], to approximately $B_{5} \approx 15 \mathrm{~T}$, when a thin film of the same material is bent [58].

The wave functions of Hamiltonian (2.1) are derived in appendix A. Their final expressions read

$$
\psi_{n=0, p_{2}, p_{3}}(\mathbf{x})=\left|e B_{\lambda}\right|^{1 / 4} e^{i p_{3} x_{3}+i p_{2} x_{2}} Y_{0}(\xi) \mathcal{P}_{s_{B}}\left(\begin{array}{l}
0 \\
1
\end{array}\right),
$$




$$
\begin{aligned}
\psi_{n>0, p_{2}, p_{3}}(\mathbf{x})= & \left|e B_{\lambda}\right|^{1 / 4} \sqrt{\frac{2 v_{F}^{2} n\left|e B_{\lambda}\right|}{2 v_{F}^{2} n\left|e B_{\lambda}\right|+\left(\epsilon_{n}-s_{B} v_{F} \lambda p_{3}\right)^{2}}} \mathcal{P}_{s_{B}} e^{i p_{3} x_{3}+i p_{2} x_{2}} \\
& \times\left\{Y_{n}(\xi)\left(\begin{array}{l}
0 \\
1
\end{array}\right)-i \frac{\lambda}{v_{F} \sqrt{2 n\left|e B_{\lambda}\right|}}\left(\epsilon_{n}-s_{B} v_{F} \lambda p_{3}\right) Y_{n-1}(\xi)\left(\begin{array}{l}
1 \\
0
\end{array}\right)\right\},
\end{aligned}
$$

where

$$
\begin{aligned}
& \epsilon_{n=0}=s_{B} v_{F} \lambda p_{3}, \\
& \epsilon_{n>0}= \pm v_{F} \sqrt{p_{3}^{2}+2 n\left|e B_{\lambda}\right|}
\end{aligned}
$$

are the energy dispersion relations at the lowest $n=0$ and higher $n>0$ Landau levels, respectively. In eqs. (2.2a) and (2.2b), we used the operator

$$
\mathcal{P}_{s_{B}}=\frac{\left(1-s_{B}\right)}{2} \sigma_{x}+\frac{\left(1+s_{B}\right)}{2},
$$

which interchanges spinor components of the wave functions when the sign $s_{B}=\operatorname{sgn}\left(e B_{\lambda}\right)$ changes. We also used the following shorthand notation:

$$
Y_{n}(\xi)=\frac{1}{\sqrt{2^{n} n ! \sqrt{\pi}}} e^{-\xi^{2} / 2} H_{n}(\xi)
$$

where $H_{n}(\xi)$ denote the Hermite polynomials and $\xi \equiv \sqrt{\left|e B_{\lambda}\right|}\left[x_{1}+p_{2} /\left(e B_{\lambda}\right)\right]$.

The Wigner function of a many-body system can be defined in terms of the second quantized fermion and antifermion fields. In a constant magnetic field, the former reads

$$
\hat{\Psi}(\mathbf{x})=\sum_{n=0}^{\infty} \int \frac{d k_{2} d k_{3}}{(2 \pi)^{2}}\left[\hat{a}_{n, k_{2}, k_{3}} \psi_{n, k_{2}, k_{3}}(\mathbf{x})+\hat{b}_{n, k_{2}, k_{3}}^{\dagger} \phi_{n, k_{2}, k_{3}}(\mathbf{x})\right]
$$

where the summation over the Landau levels and the integration over the corresponding momenta are performed. Here, $\hat{a}_{n, k_{2}, k_{3}}$ denote the particle annihilation operators and $\hat{b}_{n, k_{2}, k_{3}}^{\dagger}$ are the antiparticle creation operators. (The Hermitian conjugate field will be similarly given in terms of particle creation and antiparticle annihilation operators $\hat{a}_{n, k_{2}, k_{3}}^{\dagger}$ and $\hat{b}_{n, k_{2}, k_{3}}$, respectively.) Both sets of creation and annihilation operators satisfy the conventional anticommutator relations. While the spinors for particle states $\psi_{n, k_{2}, k_{3}}$ are given by eqs. (2.2a) and (2.2b), the spinors for antiparticles are defined by $\left.\phi_{n, k_{2}, k_{3}} \equiv \psi_{n, k_{2}, k_{3}}\right|_{\epsilon_{n} \rightarrow-\mid \epsilon_{n}}{ }$.

\section{Wigner function in a constant magnetic field}

In a relativistic theory, there are several varieties of the Wigner functions. The Lorentzcovariant forms of the Wigner function were proposed in the context of relativistic quantum statistical mechanics [59, 60], as well as in the quantum transport of QCD [36-38]. However, the covariant formulations lead to conceptual difficulties when one attempts to solve the kinetic equation as an initial-value problem. In addition, the physical interpretation of the covariant Wigner function is quite obscure. The alternative approach is to use the 
equal-time Wigner function [43] which breaks explicitly the Lorentz covariance because the Fourier transform with respect to the relative time coordinate is not performed. However, such an equal-time Wigner function poses a mathematically well-defined initial-value problem and its interpretation as a quasiprobability distribution function in the phase space is physically transparent $[43,44,61,62$ ]. [Note that the Wigner function is a quasiprobability distribution function because it can take negative values]. The situation is quite similar to the study of the Bethe-Salpeter equation which is also fully covariant, but a physical interpretation of the Bethe-Salpeter two-body wave function is rather obscure.

The equal-time Wigner operator for the Weyl fermions is given by [43]

$$
\hat{W}_{\alpha \eta}(\mathbf{x}, \mathbf{p})=\frac{1}{2} \int d^{3} \mathbf{y} e^{-i \mathbf{p} \cdot \mathbf{y}} e^{i \Phi\left(\mathbf{r}_{+}, \mathbf{r}_{-}\right)}\left[\hat{\Psi}_{\eta}^{\dagger}\left(\mathbf{r}_{+}\right), \hat{\Psi}_{\alpha}\left(\mathbf{r}_{-}\right)\right],
$$

where $\hat{\Psi}_{\alpha}$ and $\hat{\Psi}_{\eta}^{\dagger}$ are the spinor components of the chiral fermion fields given by eq. (2.6) and its Hermitian conjugated expression, respectively; $\mathbf{r}_{ \pm}=\mathbf{x} \pm \mathbf{y} / 2$, the square brackets denote the commutator, and the Schwinger phase

$$
\Phi\left(\mathbf{r}_{+}, \mathbf{r}_{-}\right)=-e \int_{\mathbf{r}_{-}}^{\mathbf{r}_{+}} d \mathbf{r} \mathbf{A}_{\lambda}(\mathbf{r})=-e B_{\lambda} y_{2} x_{1}
$$

ensures the gauge invariance of $\hat{W}_{\alpha \eta}(\mathbf{x}, \mathbf{p})$. It is worth noting that instead of the usual normal ordering, where the vacuum parts are simply omitted, we employed the Schwinger prescription with a commutator [63]. In the absence of external fields, both definitions are physically completely equivalent. However, for time-dependent electromagnetic fields, the definition of normal ordering is ambiguous. In such a case, one should use the Schwinger prescription, which defines the Wigner function correctly transforming under the charge conjugation.

To obtain a statistical description, the Wigner operator has to be appropriately averaged. In order to do this, we introduce the density matrix operator $\hat{\rho}$, which at finite chemical potential and temperature reads

$$
\hat{\rho}=\frac{1}{Z} e^{-\beta\left(\hat{H}_{\lambda}-\mu_{\lambda} \hat{N}_{\lambda}\right)},
$$

and defines the probability of the realization of a given quantum state. Here, $\beta=1 / T$ is the inverse temperature, $\hat{H}_{\lambda}$ is the second quantized Hamiltonian, $\hat{N}_{\lambda}$ is the particle number operator, and $Z$ denotes the partition function. By definition, the latter is given by

$$
\begin{aligned}
& Z=\operatorname{Tr}\left[e^{-\beta\left(\hat{H}_{\lambda}-\mu_{\lambda} \hat{N}_{\lambda}\right)}\right]=\sum_{\Phi}\left\langle\Phi\left|e^{-\beta\left[\left(\left|\epsilon_{n}\right|-\mu_{\lambda}\right) \hat{a}_{n, k_{2}, k_{3}}^{\dagger} \hat{a}_{n, k_{2}, k_{3}}-\left(-\left|\epsilon_{n}\right|-\mu_{\lambda}\right) \hat{b}_{n, k_{2}, k_{3}}^{\dagger} \hat{b}_{n, k_{2}, k_{3}}\right]}\right| \Phi\right\rangle \\
& =\prod_{n, k_{2}, k_{3}}\left(1+e^{-\beta\left(\left|\epsilon_{n}\right|-\mu_{\lambda}\right)}\right)\left(1+e^{-\beta\left(\left|\epsilon_{n}\right|+\mu_{\lambda}\right)}\right) \text {, }
\end{aligned}
$$

where $\operatorname{Tr}[\ldots]$ denotes the trace over the Hilbert space of the multi-particles states $|\Phi\rangle=$ $\left|\ldots, N_{m_{i}}, \ldots, \bar{N}_{m_{j}}, \ldots\right\rangle$ with $N_{m_{i}}$ particles in state $m_{i}$ and $\bar{N}_{m_{j}}$ antiparticles in state $m_{j}$. Note that the minus sign at the $\hat{b}_{n, k_{2}, k_{3}}^{\dagger} \hat{b}_{n, k_{2}, k_{3}}$ term in the exponent comes from the normal ordering of the anticommutating fermion operators. 
By definition, the Wigner function is the ensemble average of the Wigner operator (3.1), i.e.,

$$
W_{\alpha \eta}(\mathbf{x}, \mathbf{p}) \equiv \operatorname{Tr}\left(\hat{W}_{\alpha \eta}(\mathbf{x}, \mathbf{p}) \hat{\rho}\right) .
$$

Its detailed derivation in the case of chiral fermions in a constant external magnetic field is presented in appendix B. The final result can be conveniently given in the form of an expansion in the Pauli matrices,

$$
W(\mathbf{x}, \mathbf{p})=w_{0}(\mathbf{x}, \mathbf{p})+\lambda \sum_{i=1}^{3} \sigma_{i} w_{i}(\mathbf{x}, \mathbf{p}) .
$$

Henceforth, we will omit the arguments of $w_{0}, \mathbf{w}$, and $W$. Strictly speaking, the corresponding functions do not depend on spatial coordinates when the magnetic field is uniform. The explicit expressions for the vector components of the Wigner function $\mathbf{w}=\operatorname{tr}(\boldsymbol{\sigma} W) / 2$ are given by

$$
\begin{aligned}
w_{1}= & 2 e^{-p_{\perp}^{2} /\left|e B_{\lambda}\right|} \sum_{n=0}^{\infty} \sum_{\epsilon_{n}}(-1)^{n} \frac{v_{F} p_{1}}{\left|\epsilon_{n}\right|} L_{n-1}^{1}\left(\frac{2 p_{\perp}^{2}}{\left|e B_{\lambda}\right|}\right)\left\{\frac{\theta\left(\epsilon_{n}\right)}{1+e^{\beta\left(\left|\epsilon_{n}\right|-\mu_{\lambda}\right)}}+\frac{\theta\left(-\epsilon_{n}\right)}{1+e^{\beta\left(\left|\epsilon_{n}\right|+\mu_{\lambda}\right)}}-\frac{1}{2}\right\}, \\
w_{2}= & 2 e^{-p_{\perp}^{2} /\left|e B_{\lambda}\right|} \sum_{n=0}^{\infty} \sum_{\epsilon_{n}}(-1)^{n} \frac{v_{F} p_{2}}{\left|\epsilon_{n}\right|} L_{n-1}^{1}\left(\frac{2 p_{\perp}^{2}}{\left|e B_{\lambda}\right|}\right)\left\{\frac{\theta\left(\epsilon_{n}\right)}{1+e^{\beta\left(\left|\epsilon_{n}\right|-\mu_{\lambda}\right)}}+\frac{\theta\left(-\epsilon_{n}\right)}{1+e^{\beta\left(\left|\epsilon_{n}\right|+\mu_{\lambda}\right)}}-\frac{1}{2}\right\}, \\
w_{3}= & \lambda s_{B} \frac{e^{-p_{\perp}^{2} /\left|e B_{\lambda}\right|}}{2} \sum_{n=0}^{\infty} \sum_{\epsilon_{n}} \frac{(-1)^{n}}{\left|\epsilon_{n}\right|} \\
& \times\left\{-\left[\left(\left|\epsilon_{n}\right|+s_{B} \lambda v_{F} p_{3}\right) L_{n}\left(\frac{2 p_{\perp}^{2}}{\left|e B_{\lambda}\right|}\right)+\left(\left|\epsilon_{n}\right|-s_{B} \lambda v_{F} p_{3}\right) L_{n-1}\left(\frac{2 p_{\perp}^{2}}{\left|e B_{\lambda}\right|}\right)\right] \frac{\theta\left(\epsilon_{n}\right)}{1+e^{\beta\left(\left|\epsilon_{n}\right|-\mu_{\lambda}\right)}}\right. \\
& +\left[\left(\left|\epsilon_{n}\right|-s_{B} \lambda v_{F} p_{3}\right) L_{n}\left(\frac{2 p_{\perp}^{2}}{\left|e B_{\lambda}\right|}\right)+\left(\left|\epsilon_{n}\right|+s_{B} \lambda v_{F} p_{3}\right) L_{n-1}\left(\frac{2 p_{\perp}^{2}}{\left|e B_{\lambda}\right|}\right)\right] \frac{\theta\left(-\epsilon_{n}\right)}{1+e^{\beta\left(\left|\epsilon_{n}\right|+\mu_{\lambda}\right)}} \\
& \left.+\frac{1}{2}\left[\left(\epsilon_{n}+s_{B} \lambda v_{F} p_{3}\right) L_{n}\left(\frac{2 p_{\perp}^{2}}{\left|e B_{\lambda}\right|}\right)+\left(\epsilon_{n}-s_{B} \lambda v_{F} p_{3}\right) L_{n-1}\left(\frac{2 p_{\perp}^{2}}{\left|e B_{\lambda}\right|}\right)\right]\right\},
\end{aligned}
$$

where $p_{\perp}^{2}=p_{1}^{2}+p_{2}^{2}, \theta(x)$ denotes the unit step function, and the sum $\sum_{\epsilon_{n}}$ takes into account both positive and negative branches of the energy spectrum at $n>0$. The scalar part of the Wigner function $w_{0}=\operatorname{tr}(W) / 2$ defines the quasiprobability distribution function $f_{\mathrm{W}, \lambda}(\mathbf{p}) \equiv 2 w_{0}$. The explicit expression of the latter reads

$$
\begin{aligned}
f_{\mathrm{W}, \lambda}(\mathbf{p})= & 2 e^{-p_{\perp}^{2} /\left|e B_{\lambda}\right|}\left\{\frac{\theta\left(\epsilon_{0}\right)}{1+e^{\beta\left(\left|\epsilon_{0}\right|-\mu_{\lambda}\right)}}-\frac{\theta\left(-\epsilon_{0}\right)}{1+e^{\beta\left(\left|\epsilon_{0}\right|+\mu_{\lambda}\right)}}-\frac{\operatorname{sgn}\left(\epsilon_{0}\right)}{2}\right\}+e^{-p_{\perp}^{2} /\left|e B_{\lambda}\right|} \sum_{n=1}^{\infty} \frac{(-1)^{n}}{\left|\epsilon_{n}\right|} \\
& \times\left\{\left[\left(\left|\epsilon_{n}\right|+s_{B} \lambda v_{F} p_{3}\right) L_{n}\left(\frac{2 p_{\perp}^{2}}{\left|e B_{\lambda}\right|}\right)-\left(\left|\epsilon_{n}\right|-s_{B} \lambda v_{F} p_{3}\right) L_{n-1}\left(\frac{2 p_{\perp}^{2}}{\left|e B_{\lambda}\right|}\right)\right] \frac{1}{1+e^{\beta\left(\left|\epsilon_{n}\right|-\mu_{\lambda}\right)}}\right. \\
& -\left[\left(\left|\epsilon_{n}\right|-s_{B} \lambda v_{F} p_{3}\right) L_{n}\left(\frac{2 p_{\perp}^{2}}{\left|e B_{\lambda}\right|}\right)-\left(\left|\epsilon_{n}\right|+s_{B} \lambda v_{F} p_{3}\right) L_{n-1}\left(\frac{2 p_{\perp}^{2}}{\left|e B_{\lambda}\right|}\right)\right] \frac{1}{1+e^{\beta\left(\left|\epsilon_{n}\right|+\mu_{\lambda}\right)}} \\
& \left.-s_{B} \lambda v_{F} p_{3}\left[L_{n}\left(\frac{2 p_{\perp}^{2}}{\left|e B_{\lambda}\right|}\right)+L_{n-1}\left(\frac{2 p_{\perp}^{2}}{\left|e B_{\lambda}\right|}\right)\right]\right\} .
\end{aligned}
$$



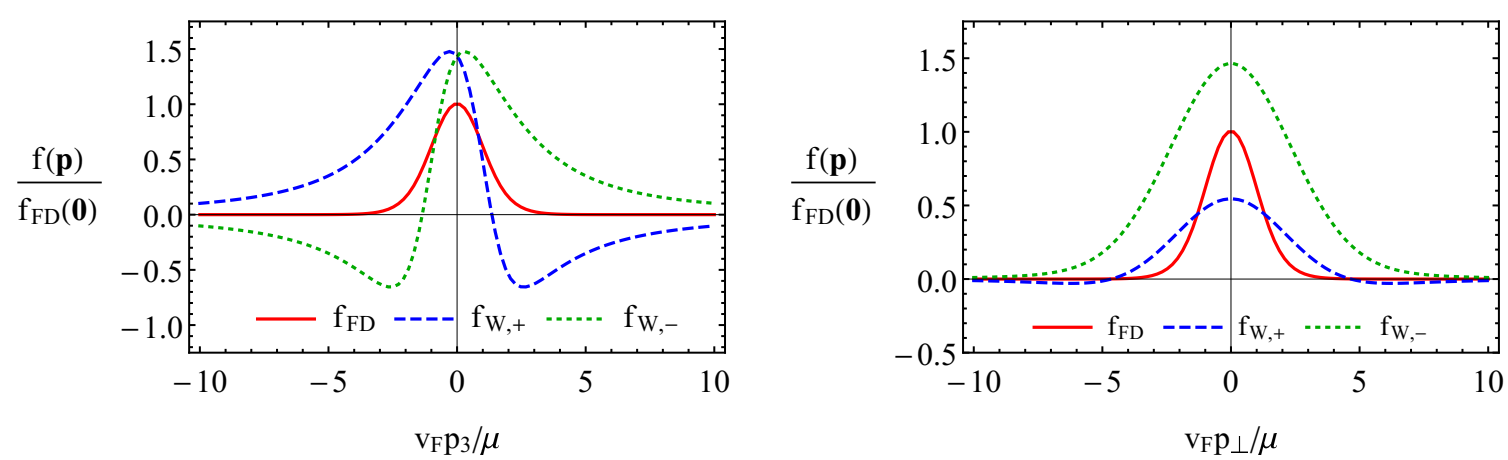

Figure 1. The dependence of the normalized Wigner quasiprobability distribution functions on $p_{3}$ (left panel) and $p_{\perp}$ (right panel) at $B_{\lambda}=0$ (red solid lines) and $B_{\lambda} \neq 0$ (blue dashed and green doted lines correspond to the right-handed and left-handed fermions, respectively). In the left panel $p_{\perp}=\mu / v_{F}$ and $p_{3}=\mu / v_{F}$ in the right panel. The other parameters are set as follows: $\mu_{5}=0$, $T=0.5 \mu, B_{5}=0$, and $e B=10\left(\mu / v_{F}\right)^{2}$.

In order to get a better insight into the Wigner quasiprobability distribution function $f_{\mathrm{W}, \lambda}(\mathbf{p})$, it is instructive to compare it with the standard quasiprobability $f_{\mathrm{FD}}(\mathbf{p})$ at $B_{\lambda}=$ 0 , which is given in terms of the Fermi-Dirac functions, i.e.,

$$
f_{\mathrm{FD}}(\mathbf{p})=n_{\mathrm{F}}\left(\epsilon_{\mathbf{p}}-\mu_{\lambda}\right)-n_{\mathrm{F}}\left(\epsilon_{\mathbf{p}}+\mu_{\lambda}\right)
$$

where $\epsilon_{\mathbf{p}}=v_{F}|\mathbf{p}|$ and $n_{\mathrm{F}}(x)=1 /\left(e^{\beta x}+1\right)$ is the Fermi-Dirac distribution function. The numerical comparison of the two quasiprobability distribution functions is shown in figure 1. As we can see, the inclusion of the magnetic field leads to several qualitative changes in the dependence of the quasiprobabilities on the longitudinal and transverse momenta, presented in the left and right panels of figure 1, respectively. While the quasiprobability function (3.9) is always positive (assuming $\mu_{\lambda}>0$ ), its counterpart in the background magnetic field takes negative values in a range of momenta. Such negative values of the quasiprobability originate from the quantum effects that cannot be captured by usual distribution functions. As is seen from the left panel in figure 1, the dependence of the quasiprobability distribution function $f_{\mathrm{W}, \lambda}(\mathbf{p})$ on $p_{3}$ is asymmetric in the longitudinal component of momentum $p_{3}$, as well as in chirality. A chiral asymmetry is also clearly visible in the right panel in figure 1 , where the distributions $f_{\mathrm{W}, \pm}(\mathbf{p})$ have different widths and heights as functions of $p_{\perp}$.

Further, we plot the dependence of the vector component of the Wigner function along the magnetic field $w_{3}$ on $p_{3}$ and $p_{\perp}$ in the left and right panels of figure 2 , respectively. Similarly to the Wigner quasiprobability distribution function, the corresponding dependence is also asymmetric with respect to $p_{3}$ and the chirality. Note that the asymmetry is well-pronounced at small values of momenta.

Before proceeding further with the analysis of the Wigner function in a strong magnetic field, we will test our results (3.6)-(3.8) by studying the chiral magnetic effect (CME) and chiral separation effect (CSE), as well as deriving the weak-field limit of the function $W$. 

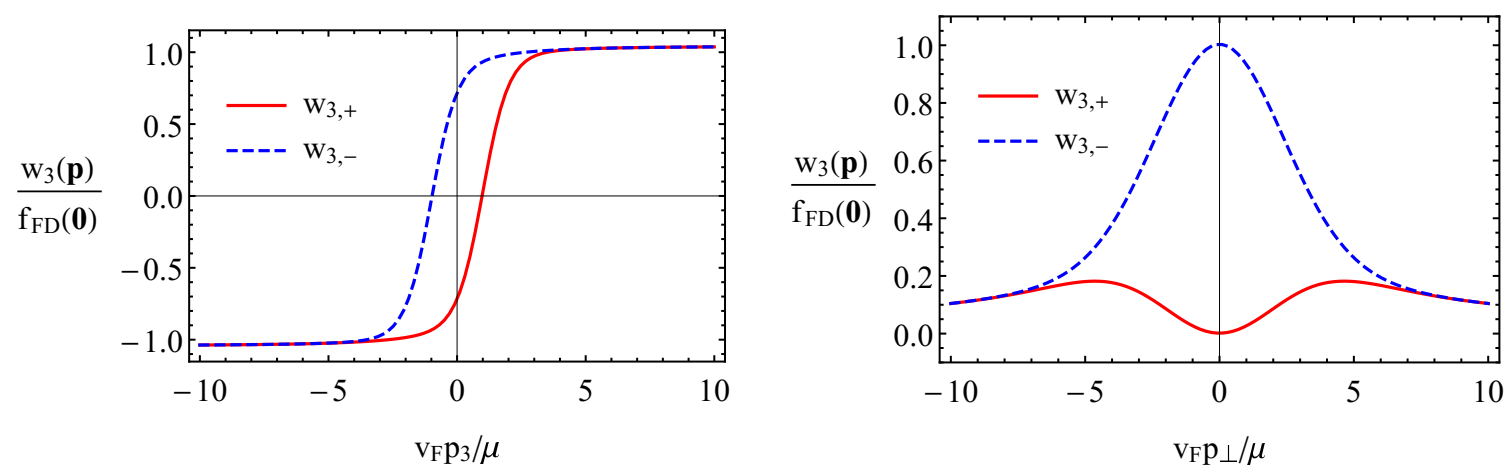

Figure 2. The dependence of the normalized vector component of the Wigner function along the magnetic field defined by eq. (3.7c) on $p_{3}$ (left panel) and $p_{\perp}$ (right panel). Red solid and blue dashed lines correspond to the right-handed and left-handed fermions, respectively. In the left panel $p_{\perp}=\mu / v_{F}$ and $p_{3}=\mu / v_{F}$ in the right panel. The other parameters are set as follows: $\mu_{5}=0$, $T=0.5 \mu, B_{5}=0$, and $e B=10\left(\mu / v_{F}\right)^{2}$.

\subsection{Chiral magnetic and chiral separation effects}

We begin our analysis of the Wigner function (3.6) with the study of the chiral magnetic and chiral separation effects. The electric and chiral current densities are defined by

$$
\begin{aligned}
\mathbf{j} & \equiv-e v_{F} \sum_{\lambda= \pm} \lambda \int \frac{d^{3} \mathbf{p}}{(2 \pi)^{3}} \operatorname{tr}[\boldsymbol{\sigma} W]=-2 e v_{F} \sum_{\lambda= \pm} \int \frac{d^{3} \mathbf{p}}{(2 \pi)^{3}} \mathbf{w} \\
\mathbf{j}_{5} & \equiv-e v_{F} \sum_{\lambda= \pm} \int \frac{d^{3} \mathbf{p}}{(2 \pi)^{3}} \operatorname{tr}[\boldsymbol{\sigma} W]=-2 e v_{F} \sum_{\lambda= \pm} \lambda \int \frac{d^{3} \mathbf{p}}{(2 \pi)^{3}} \mathbf{w} .
\end{aligned}
$$

It is worth noting that the factor $\lambda$ in eq. (3.10a) comes from the definition of the electric current operator

$$
j_{i, \lambda}(\mathbf{p}) \equiv-e\left(\partial_{p_{i}} H_{\lambda}\right)=-e \lambda v_{F} \sigma_{i}
$$

Taking into account the Wigner function components given by eqs. (3.7a)-(3.7c) one can easily see that the only nonzero component of the current is along the $\hat{\mathbf{z}}$ direction. Integrating over $p_{\perp}$, we obtain

$$
\begin{aligned}
j_{3, \lambda}= & \lambda e v_{F} \frac{e B_{\lambda}}{(2 \pi)^{2}} \int d p_{3}\left[\frac{\theta\left(s_{B} \lambda v_{F} p_{3}\right)}{1+e^{\beta\left(v_{F}\left|p_{3}\right|-\mu_{\lambda}\right)}}-\frac{\theta\left(-s_{B} \lambda v_{F} p_{3}\right)}{1+e^{\beta\left(v_{F}\left|p_{3}\right|+\mu_{\lambda}\right)}}-s_{B} \lambda \frac{\operatorname{sgn}\left(p_{3}\right)}{2}\right] \\
& +e v_{F} \frac{\left|e B_{\lambda}\right|}{(2 \pi)^{2}} \sum_{n=1}^{\infty} \int d p_{3} \frac{p_{3}}{\sqrt{p_{3}^{2}+2 n\left|e B_{\lambda}\right|}}\left[\frac{\theta\left(\epsilon_{n}\right)}{1+e^{\beta\left(\left|\epsilon_{n}\right|-\mu_{\lambda}\right)}}+\frac{\theta\left(-\epsilon_{n}\right)}{1+e^{\beta\left(\left|\epsilon_{n}\right|+\mu_{\lambda}\right)}}-\frac{1}{2}\right] \\
= & \lambda e \frac{e B_{\lambda}}{(2 \pi)^{2}} \int d p_{3} T\left[\ln \left(1+e^{\beta \mu_{\lambda}}\right)-\ln \left(1+e^{-\beta \mu_{\lambda}}\right)\right]=\lambda \frac{e^{2} B_{\lambda} \mu_{\lambda}}{(2 \pi)^{2}},
\end{aligned}
$$

where the contribution from the higher Landau levels is zero due to the integration over $p_{3}$. Performing the summation over chiralities, we find the following standard electric and 
chiral current densities $[31,56,64,65]$ :

$$
\begin{gathered}
j_{3}=\sum_{\lambda} j_{3, \lambda}=\frac{e^{2} B \mu_{5}}{2 \pi^{2}}+\frac{e^{2} B_{5} \mu}{2 \pi^{2}}, \\
j_{3,5}=\sum_{\lambda} \lambda j_{3, \lambda}=\frac{e^{2} B \mu}{2 \pi^{2}}+\frac{e^{2} B_{5} \mu_{5}}{2 \pi^{2}} .
\end{gathered}
$$

Thus, as expected, we reproduce exactly the standard relations for the CSE and CME using the Wigner function approach.

\subsection{Weak magnetic field expansion}

In this subsection we consider the limit of small magnetic fields $\left|e B_{\lambda}\right| \ll p^{2}$. After expanding the Wigner function to the linear order in $\left|e B_{\lambda}\right| / p^{2}$ and performing the summation over the Landau levels (see appendix $\mathrm{C}$ for details), we arrive at the following expressions for the scalar $w_{0}$ and vector $\mathbf{w}$ parts:

$$
\begin{aligned}
w_{0}= & \frac{1}{2}\left[n_{\mathrm{F}}\left(\epsilon_{\mathbf{p}}-\mu_{\lambda}\right)-n_{\mathrm{F}}\left(\epsilon_{\mathbf{p}}+\mu_{\lambda}\right)\right] \\
& -\frac{\lambda v_{F}^{2} e B_{\lambda}}{4} \frac{v_{F} p_{3}}{\epsilon_{\mathbf{p}}} \frac{d}{d \epsilon_{\mathbf{p}}} \frac{1}{\epsilon_{\mathbf{p}}}\left[n_{\mathrm{F}}\left(\epsilon_{\mathbf{p}}-\mu_{\lambda}\right)+n_{\mathrm{F}}\left(\epsilon_{\mathbf{p}}+\mu_{\lambda}\right)-1\right]+O\left(\left|e B_{\lambda}\right|^{2}\right), \\
w_{j}= & -\frac{v_{F} p_{j}}{2} \frac{1}{\epsilon_{\mathbf{p}}}\left[n_{\mathrm{F}}\left(\epsilon_{\mathbf{p}}-\mu_{\lambda}\right)+n_{\mathrm{F}}\left(\epsilon_{\mathbf{p}}+\mu_{\lambda}\right)-1\right] \\
& +\frac{\lambda v_{F}^{2} e\left(B_{\lambda}\right)_{j}}{4} \frac{1}{\epsilon_{\mathbf{p}}} \frac{d}{d \epsilon_{\mathbf{p}}}\left[n_{\mathrm{F}}\left(\epsilon_{\mathbf{p}}-\mu_{\lambda}\right)-n_{\mathrm{F}}\left(\epsilon_{\mathbf{p}}+\mu_{\lambda}\right)\right]+O\left(\left|e B_{\lambda}\right|^{2}\right) .
\end{aligned}
$$

The above expressions qualitatively agree with the results obtained in refs. [42, 66, 67]. Note, however, that those papers use the covariant definition of the Wigner function. Therefore, one needs to integrate their results over the zeroth component of the fourmomentum $p_{0}$ before comparing with eqs. (3.15a) and (3.15b). It is interesting to note that we have the additional term -1 in the second square brackets of eq. (3.15a) as well

as in the first square brackets of eq. (3.15b), which is absent in refs. [42, 66, 67]. This difference is connected with our use of the commutator in the definition of the Wigner operator (3.1) instead of the usual normal ordering considered in the cited works.

By making use of the explicit expressions for the Fermi-Dirac distribution functions, the results for the scalar and vector parts of the Wigner function can be rewritten in the following equivalent form:

$$
\begin{aligned}
w_{0} \simeq \frac{1}{2}\{ & {\left[1+e\left(\mathbf{B}_{\lambda} \cdot \boldsymbol{\Omega}_{\lambda}\right)\right] n_{\mathrm{F}}\left(\epsilon_{\mathbf{p}}-\frac{\lambda e v_{F}\left(\mathbf{B}_{\lambda} \cdot \mathbf{p}\right)}{2|\mathbf{p}|^{2}}-\mu_{\lambda}\right) } \\
& \left.\quad-\left[1-e\left(\mathbf{B}_{\lambda} \cdot \boldsymbol{\Omega}_{\lambda}\right)\right] n_{\mathrm{F}}\left(\epsilon_{\mathbf{p}}+\frac{\lambda e v_{F}\left(\mathbf{B}_{\lambda} \cdot \mathbf{p}\right)}{2|\mathbf{p}|^{2}}+\mu_{\lambda}\right)\right\}-\frac{e\left(\mathbf{B}_{\lambda} \cdot \boldsymbol{\Omega}_{\lambda}\right)}{2}+O\left(\left|e B_{\lambda}\right|^{2}\right),
\end{aligned}
$$

where $\boldsymbol{\Omega}_{\lambda}=\lambda \mathbf{p} /\left(2|\mathbf{p}|^{3}\right)$ is the Berry curvature. This result agrees with the distribution function in the chiral kinetic theory, except for the last term, which originates from the 
commutator in the definition of the Wigner operator (3.1). Note that due to the presence of the Berry curvature and magnetic field, the quasiprobability distribution function in eq. (3.16) can take negative values when the magnetic field is nonzero.

\section{Equation of motion for the Wigner function}

In this section we present the equation of motion for the equal-time Wigner function in external electromagnetic fields. According to ref. [43], the corresponding equation reads

$$
D_{0} W+v_{F} \frac{\lambda}{2} \mathbf{D} \cdot\{\boldsymbol{\sigma}, W\}-i \lambda v_{F}[(\boldsymbol{\sigma} \cdot \mathbf{P}), W]=0 .
$$

Here $\{$,$\} and [,] denote anticommutator and commutator, respectively, and the following$ derivatives are used:

$$
\begin{aligned}
D_{0} & \equiv \partial_{t}+e \int_{-1 / 2}^{1 / 2} d s\left(\mathbf{E}\left(\mathbf{r}+i s \partial_{\mathbf{p}}\right) \cdot \partial_{\mathbf{p}}\right) \approx \partial_{t}+e\left(\mathbf{E}(\mathbf{r}) \cdot \partial_{\mathbf{p}}\right)+O\left[\left(\nabla_{\mathbf{r}} \cdot \partial_{\mathbf{p}}\right) \mathbf{E}(\mathbf{r})\right], \\
\mathbf{D} & \equiv \partial_{\mathbf{r}}+e \int_{-1 / 2}^{1 / 2} d s\left[\mathbf{B}\left(\mathbf{r}+i s \partial_{\mathbf{p}}\right) \times \partial_{\mathbf{p}}\right] \approx \partial_{\mathbf{r}}+e\left[\mathbf{B}(\mathbf{r}) \times \partial_{\mathbf{p}}\right]+O\left[\left(\nabla_{\mathbf{r}} \times \partial_{\mathbf{p}}\right) \mathbf{B}(\mathbf{r})\right], \\
\mathbf{P} & \equiv \mathbf{p}+i e \int_{-1 / 2}^{1 / 2} d s s\left[\mathbf{B}\left(\mathbf{r}+i s \partial_{\mathbf{p}}\right) \times \partial_{\mathbf{p}}\right] \approx \mathbf{p}+O\left[\left(\nabla_{\mathbf{r}} \cdot \partial_{\mathbf{p}}\right) \mathbf{B}(\mathbf{r})\right] .
\end{aligned}
$$

As one can see from the above equations, the derivatives become local when the external fields are spatially uniform. In terms of the scalar $w_{0}$ and vector $\mathbf{w}$ parts of the Wigner function, eq. (4.1) reads

$$
\begin{aligned}
D_{0} w_{0}+v_{F}(\mathbf{D} \cdot \mathbf{w}) & =0, \\
D_{0} \mathbf{w}+v_{F} \mathbf{D} w_{0}+2 \lambda v_{F}[\mathbf{P} \times \mathbf{w}] & =0 .
\end{aligned}
$$

In the next section, we will use eqs. (4.3a) and (4.3b) to study the longitudinal modes of the chiral magnetic wave (CMW) [68] in the limit of a strong magnetic field. In order to describe such a collective excitation taking into account the dynamical electromagnetism, we consider the system subjected to small oscillating electromagnetic fields

$$
\begin{aligned}
& \mathbf{E}^{\prime}=\mathbf{E} e^{-i \omega t+i \mathbf{k} \cdot \mathbf{r}} \\
& \mathbf{B}^{\prime}=\mathbf{B} e^{-i \omega t+i \mathbf{k} \cdot \mathbf{r}}
\end{aligned}
$$

and a strong constant effective magnetic field $\mathbf{B}_{0, \lambda}$. [In the case of a weak magnetic field, the effects of the dynamical electromagnetism were taken into account in refs. [69, 70].] In this case, the Wigner function can be naturally split in two parts, i.e., $W=W^{(0)}+W^{\prime}$. While the first part corresponds to the constant external magnetic field $\mathbf{B}_{0, \lambda}$, the second one is related to the oscillating fields. The latter can be written in the form

$$
W^{\prime}=W^{(1)} e^{-i \omega t+i \mathbf{k} \cdot \mathbf{r}}=w_{0}^{\prime}+\lambda\left(\boldsymbol{\sigma} \cdot \mathbf{w}^{\prime}\right)=w_{0}^{(1)} e^{-i \omega t+i \mathbf{k} \cdot \mathbf{r}}+\lambda\left(\boldsymbol{\sigma} \cdot \mathbf{w}^{(1)}\right) e^{-i \omega t+i \mathbf{k} \cdot \mathbf{r}} .
$$


To the linear order in the oscillating electromagnetic fields, the components of the Wigner function satisfy the following equations:

$$
\begin{array}{r}
\partial_{t} w_{0}^{\prime}+v_{F} \partial_{\mathbf{r}} \mathbf{w}^{\prime}+v_{F} e\left(\left[\mathbf{B}_{0, \lambda} \times \partial_{\mathbf{p}}\right] \cdot \mathbf{w}^{\prime}\right)+e\left(\mathbf{E}^{\prime} \cdot \partial_{\mathbf{p}}\right) \int_{-1 / 2}^{1 / 2} d s w_{0}^{(0)}(\mathbf{p}-s \mathbf{k}) \\
+v_{F} e\left[\mathbf{B}^{\prime} \times \partial_{\mathbf{p}}\right] \int_{-1 / 2}^{1 / 2} d s \mathbf{w}^{(0)}(\mathbf{p}-s \mathbf{k})=0, \\
\partial_{t} \mathbf{w}^{\prime}+v_{F} \partial_{\mathbf{r}} w_{0}^{\prime}+v_{F} e\left[\mathbf{B}_{0, \lambda} \times \partial_{\mathbf{p}}\right] w_{0}^{\prime}+2 \lambda v_{F}\left[\mathbf{p} \times \mathbf{w}^{\prime}\right] \\
-v_{F} e\left[\mathbf{B}^{\prime} \times \partial_{\mathbf{p}}\right] \int_{-1 / 2}^{1 / 2} d s w_{0}^{(0)}(\mathbf{p}-s \mathbf{k})+e\left(\mathbf{E}^{\prime} \cdot \partial_{\mathbf{p}}\right) \int_{-1 / 2}^{1 / 2} d s \mathbf{w}^{(0)}(\mathbf{p}-s \mathbf{k}) \\
+2 i e \lambda v_{F} \int_{-1 / 2}^{1 / 2} d s s\left[\left[\mathbf{B}^{\prime} \times \partial_{\mathbf{p}}\right] \times \mathbf{w}^{(0)}(\mathbf{p}-s \mathbf{k})\right]=0 .
\end{array}
$$

In order to find the spectrum of collective modes, we should determine the electric current density $j^{\prime m}$ which enters the Maxwell's equations through the polarization vector

$$
P^{\prime m}=i \frac{j^{\prime m}}{\omega}=\chi^{m n} E^{\prime n}
$$

where $\chi^{m n}(m, n=1,2,3$ denote spatial components) is the susceptibility tensor. Then, as is easy to check, the Maxwell's equations admit a nontrivial solution when the following characteristic equation is satisfied:

$$
\operatorname{det}\left[\left(\omega^{2}-k^{2}\right) \delta^{m n}+k^{m} k^{n}+4 \pi \omega^{2} \chi^{m n}\right]=0 .
$$

The solution to this equation determines the dispersion relation of electromagnetic collective modes, such as the chiral magnetic wave.

As we saw in the previous section, the scalar part of the Wigner function is related to the distribution function of the chiral kinetic theory in the limit of weak magnetic field. However, the Wigner function is applicable even beyond the weak-field limit. It is instructive, therefore, to consider the case of a strong constant magnetic field $\mathbf{B}_{0, \lambda} \| \hat{\mathbf{z}}$. In such a limit, one can use the lowest Landau level (LLL) approximation when only the LLL contribution is retained. Then, the scalar and vector components of the Wigner function take the form

$$
\begin{aligned}
w_{0}^{(0)} & =e^{-p_{\perp}^{2} /\left|e B_{0, \lambda}\right|} \tilde{f}_{\mathrm{LLL}}\left(p_{3}\right), \\
\mathbf{w}^{(0)} & =-s_{B} \lambda \hat{\mathbf{z}} e^{-p_{\perp}^{2} /\left|e B_{0, \lambda}\right|} \tilde{f}_{\mathrm{LLL}}\left(p_{3}\right),
\end{aligned}
$$

where

$\tilde{f}_{\mathrm{LLL}}\left(p_{3}\right) \equiv \theta\left(s_{B} \lambda v_{F} p_{3}\right) n_{\mathrm{F}}\left(s_{B} \lambda v_{F} p_{3}-\mu_{\lambda}\right)-\theta\left(-s_{B} \lambda v_{F} p_{3}\right) n_{\mathrm{F}}\left(-s_{B} \lambda v_{F} p_{3}+\mu_{\lambda}\right)-\frac{\lambda s_{B} \operatorname{sgn}\left(p_{3}\right)}{2}$.

Note that both scalar and vector parts of the Wigner function are expressed in terms of the distribution function on the LLL. The last term in eq. (4.10) is related to our use of the commutator in the Wigner operator (3.1) and properly describes the vacuum oscillations. 
As we will see below, it is crucial for the correct description of the collective excitations and transport phenomena in a strong field limit.

In the next two sections, we will use the equal-time Wigner function in the LLL approximation in order to study: (i) the dispersion relation of the CMW and (ii) the thermoelectric properties of chiral fermions in a strong magnetic field.

\section{The chiral magnetic and pseudomagnetic waves in a strong magnetic field}

In this section, we study the dispersion relation of the CMW in the strong magnetic field limit by using the equal-time Wigner function approach. In order to simplify the analysis, we will consider only the case of longitudinal waves, propagating along the direction of the background field. In other words, the perpendicular components of the wave vector and the oscillating electric field will vanish, i.e., $\mathbf{k}_{\perp}=0$ and $\mathbf{E}_{\perp}^{\prime}=0$. In view of the Maxwell equations, there will be also no oscillating magnetic field, i.e., $\mathbf{B}^{\prime}=\left[\mathbf{k} \times \mathbf{E}^{\prime}\right] / \omega=0$. In this case, eqs. (4.6a) and (4.6b) reduce to

$$
\begin{array}{r}
-i \omega w_{0}^{(1)}+i v_{F} k_{3} w_{3}^{(1)}+e E_{3} e^{-p_{\perp}^{2} /\left|e B_{0, \lambda}\right|} \int_{-1 / 2}^{1 / 2} d s \partial_{p_{3}} \tilde{f}_{\mathrm{LLL}}\left(p_{3}-s k_{3}\right)=0 \\
-i \omega w_{3}^{(1)}+i v_{F} k_{3} w_{0}^{(1)}-s_{B} \lambda e E_{3} e^{-p_{\perp}^{2} /\left|e B_{0, \lambda}\right|} \int_{-1 / 2}^{1 / 2} d s \partial_{p_{3}} \tilde{f}_{\mathrm{LLL}}\left(p_{3}-s k_{3}\right)=0 .
\end{array}
$$

Note that due to the one-dimensional nature of the LLL, the equations for the $w_{1}^{(1)}$ and $w_{2}^{(1)}$ have only the trivial solutions $w_{1}^{(1)}=w_{2}^{(1)}=0$. On the other hand, the solution to the system of coupled equations (5.1a) and (5.1b) is nontrivial, i.e.,

$$
\begin{aligned}
& w_{0}^{(1)}=-i e E_{3} e^{-p_{\perp}^{2} /\left|e B_{0, \lambda}\right|} \int_{-1 / 2}^{1 / 2} d s \frac{\partial_{p_{3}} \tilde{f}_{\mathrm{LLL}}\left(p_{3}-s k_{3}\right)}{\omega+\lambda s_{B} v_{F} k_{3}} \\
& w_{3}^{(1)}=s_{B} \lambda i e E_{3} e^{-p_{\perp}^{2} /\left|e B_{0, \lambda}\right|} \int_{-1 / 2}^{1 / 2} d s \frac{\partial_{p_{3}} \tilde{f}_{\mathrm{LLL}}\left(p_{3}-s k_{3}\right)}{\omega+\lambda s_{B} v_{F} k_{3}} .
\end{aligned}
$$

By making use of the definition in eq. (3.10a), we then derive the following correction to the electric current density proportional to the oscillating electric field:

$$
j_{3}^{\prime}=-2 i e^{2} v_{F} \sum_{\lambda= \pm} s_{B} \lambda E_{3}^{\prime} \int \frac{d^{3} \mathbf{p}}{(2 \pi)^{3}} \frac{e^{-p_{\perp}^{2} /\left|e B_{0, \lambda}\right|}}{\omega+\lambda s_{B} v_{F} k_{3}} \int_{-1 / 2}^{1 / 2} d s \partial_{p_{3}} \tilde{f}_{\mathrm{LLL}}\left(p_{3}-s k_{3}\right) .
$$

It should be noted that there is also a non-oscillating contribution to the current that comes from the zeroth order Wigner function $w_{3}^{(0)}$. It describes the chiral magnetic and chiral separation effects, but does not affect directly the dispersion relation of the collective modes.

In order to perform the integral over $s$ on the right-hand side of eq. (5.3) it is convenient to rewrite the partial derivative with respect to $p_{3}$ in terms of the partial derivative with 
respect to $s$, i.e.,

$$
\begin{aligned}
I_{k_{3}} & \equiv \int_{-\Lambda}^{\Lambda} d p_{3} \int_{-1 / 2}^{1 / 2} d s \partial_{p_{3}} \tilde{f}_{\mathrm{LLL}}\left(p_{3}-s k_{3}\right)=\int_{-\Lambda}^{\Lambda} d p_{3} \int_{-1 / 2}^{1 / 2} d s \frac{1}{-k_{3}} \partial_{s} \tilde{f}_{\mathrm{LLL}}\left(p_{3}-s k_{3}\right) \\
& =2 s_{B} \lambda+\frac{2 T}{s_{B} \lambda v_{F} k_{3}}\left\{\theta\left(s_{B} \lambda\right) \frac{-s_{B} \lambda v_{F} k_{3}}{T}+\theta\left(-s_{B} \lambda\right) \frac{s_{B} \lambda v_{F} k_{3}}{T}\right\}-s_{B} \lambda \\
& =-s_{B} \lambda .
\end{aligned}
$$

Then, the final result for the electric current density (5.3) reads

$$
\begin{aligned}
j_{3}^{\prime} & =-2 i e^{2} v_{F} \sum_{\lambda= \pm} \lambda s_{B} e E_{3}^{\prime} \int \frac{d^{2} \mathbf{p}_{\perp} e^{-p_{\perp}^{2} /\left|e B_{0, \lambda}\right|}}{(2 \pi)^{3}} \frac{I_{k_{3}}}{\omega+\lambda s_{B} v_{F} k_{3}} \\
& =i e^{2} v_{F} \sum_{\lambda= \pm} \frac{E_{3}^{\prime}}{\omega+\lambda s_{B} v_{F} k_{3}} \frac{\left|e B_{0, \lambda}\right|}{(2 \pi)^{2}} .
\end{aligned}
$$

Let us first consider the case of an ordinary magnetic field background, i.e., $B_{0} \neq 0$ but $B_{0,5}=0$. By comparing with eq. (4.7), we extract the following susceptibility tensor:

$$
\chi^{33}=-\frac{v_{F} e^{2}\left|e B_{0}\right|}{2 \pi^{2}\left(\omega^{2}-v_{F}^{2} k_{3}^{2}\right)} .
$$

By substituting this into the characteristic equation (4.8), we then derive the following positive-energy solution for collective modes:

$$
\omega=\sqrt{v_{F}^{2} k_{3}^{2}+\frac{2 v_{F} e^{2}\left|e B_{0}\right|}{\pi}} .
$$

This frequency corresponds to a chiral magnetic plasmon or, equivalently, the CMW in the strong-field limit. As we see, the background magnetic field $B_{0}$ is responsible for the generation of the plasmon gap,

$$
\Omega_{B_{0}}=\sqrt{\frac{2 v_{F} e^{2}\left|e B_{0}\right|}{\pi}}
$$

We note that the above value of the gap agrees with the one obtained in the LLL approximation by a different method in ref. [71]. This is also consistent with the mass of the resonance-like photon state revealed in QED in a strong magnetic field that is realized in the kinematic regime $m^{2} \ll k_{3}^{2} \ll|e B|[72]$.

Further, let us study the case when there is only an axial magnetic field present, i.e., $B_{0}=0$ but $B_{0,5} \neq 0$. In this case, the susceptibility tensor reads

$$
\chi^{33}=-\frac{v_{F} e^{2}\left|e B_{0,5}\right|}{2 \omega \pi^{2}\left(\omega+s_{B} v_{F} k_{3}\right)} .
$$

By making use of the characteristic equation (4.8), we then derive the following dispersion relation of collective modes:

$$
\omega=\frac{1}{2}\left(\sqrt{v_{F}^{2} k_{3}^{2}+\frac{8 v_{F} e^{2}\left|e B_{0,5}\right|}{\pi}}-s_{B} v_{F} k_{3}\right) .
$$




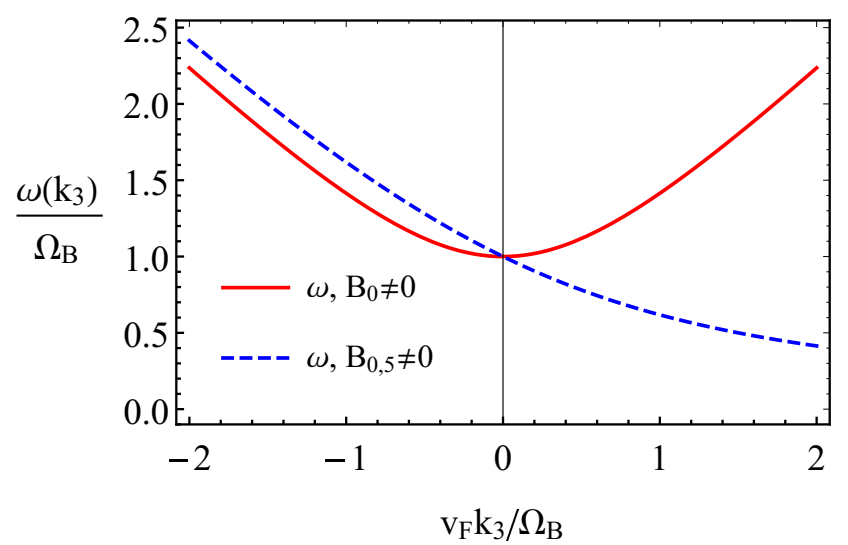

Figure 3. The frequencies of the collective excitations in the presence of background magnetic (red solid line) and axial magnetic (blue dashed line) fields, as given by eq. (5.7) and eq. (5.10), respectively. Here $\Omega_{B}=\Omega_{B_{0}}$ at $B_{0} \neq 0$ and $\Omega_{B}=\Omega_{B_{0,5}}$ at $B_{0,5} \neq 0$.

By analogy with the CMW, we call this mode a chiral pseudomagnetic wave. The dispersion relations for both types of collective modes (5.7) and (5.10) are plotted in figure 3 as functions of the longitudinal wave vector $k_{3}$. The results for a nonzero ordinary magnetic field $B_{0}$ (at $\left.B_{0,5}=0\right)$ and a nonzero axial magnetic field $B_{0,5}\left(\right.$ at $\left.B_{0}=0\right)$ are shown by solid red and blue dashed lines, respectively. Note that, the asymmetry of the dispersion relation of the chiral pseudomagnetic wave is correlated with the sign of $e B_{0,5}$. In figure 3 , we plotted the results for $e B_{0,5}>0$. The results for $e B_{0,5}<0$ can be obtained simply by replacing $k_{3} \rightarrow-k_{3}$.

As we can see from figure 3 , the chiral pseudomagnetic wave, which is realized in the case of a nonzero axial magnetic field $B_{0,5} \neq 0$, is qualitatively different from the gapped chiral magnetic wave in the presence of a usual magnetic field. Indeed, while the frequency of the chiral pseudomagnetic wave takes a nonzero value $\Omega_{B_{0,5}}=\sqrt{2 v_{F} e^{2}\left|e B_{0,5}\right| / \pi}$ at $k_{3}=0$, its dependence on the wave vector $k_{3}$ is asymmetric. The corresponding mode appears to be gapless in the strong-field limit because $\omega \rightarrow 0$ at large positive $k_{3}$ when $e B_{0,5}>0$ (or large negative $k_{3}$ when $e B_{0,5}<0$ ). We argue that the gaplessness of this mode is an artifact of the LLL approximation, which may be formally viewed as the $B_{0,5} \rightarrow \infty$ limit. In the case of a strong but finite axial magnetic field, the inclusion of higher Landau levels should make the corresponding mode gapped with the minimum of the energy obtained at $k_{3} \sim B_{0,5}$. Indeed, this would be consistent with the weak-field analysis in refs. [69, 70], where such an asymmetric dispersion relation with a minimum at $k_{3} \sim B_{0,5}$ was predicted. The solution in eq. (5.10) is nothing else, but a strong-field version of the chiral pseudomagnetic wave that was first obtained in refs. [34, 69, 70].

\section{Thermoelectric phenomena in a strong magnetic field}

In this section, we study the thermoelectric transport in a chiral plasma in a strong magnetic field. In essence, the problem reduces to determining the corrections to the Wigner function in the LLL approximation and calculating the electric and heat (thermal) cur- 
rents when an additional weak electric field $\mathbf{E}$ and a small temperature gradient $\boldsymbol{\nabla} T \neq 0$ are present. [We will take into account only the contribution due to charged chiral particles leaving aside all other possible contributions.] For simplicity, we will assume that $\mathbf{B}_{0, \lambda}\|\mathbf{E}\| \boldsymbol{\nabla} T \| \hat{\mathbf{z}}$. In this case, the equations for the Wigner function components (4.3a) and $(4.3 \mathrm{~b}) \mathrm{read}$

$$
\begin{array}{r}
\partial_{t} w_{0}+v_{F}(\boldsymbol{\nabla} \cdot \mathbf{w})+e v_{F}\left(\left[\mathbf{B}_{0, \lambda} \times \partial_{\mathbf{p}}\right] \cdot \mathbf{w}\right)+e\left(\mathbf{E} \cdot \partial_{\mathbf{p}}\right) w_{0}^{(0)}=0, \\
\partial_{t} \mathbf{w}+v_{F} \boldsymbol{\nabla} w_{0}+e v_{F}\left[\mathbf{B}_{0, \lambda} \times \partial_{\mathbf{p}}\right] w_{0}+2 \lambda v_{F}[\mathbf{p} \times \mathbf{w}]+e\left(\mathbf{E} \cdot \partial_{\mathbf{p}}\right) \mathbf{w}^{(0)}=0 .
\end{array}
$$

Because of the spatial gradient of temperature, the Wigner function depends on spatial coordinates, i.e., $\boldsymbol{\nabla} W=(\boldsymbol{\nabla} T) \partial_{T} W \approx(\boldsymbol{\nabla} T) \partial_{T} W^{(0)}$. Here we assume that the gradient $\nabla T$ is small and of the same order of magnitude as $\mathbf{E}$.

By using the Wigner function components calculated in the LLL approximation, see eqs. (4.9a) and (4.9b), as well as introducing a phenomenological collision term in the relaxation time approximation on the right-hand sides of eqs. (6.1a) and (6.1b), we obtain the following set of equations:

$$
\begin{aligned}
& e^{-p_{\perp}^{2} /\left|e B_{0, \lambda}\right|}\left[\left(-s_{B} \lambda\right) v_{F}\left(\nabla_{3} T\right) \partial_{T}+e E_{3} \partial_{p_{3}}\right] \tilde{f}_{L L L}\left(p_{3}\right)=-\frac{w_{0}-e^{-p_{\perp}^{2} /\left|e B_{0, \lambda}\right|} \tilde{f}_{\mathrm{LLL}}\left(p_{3}\right)}{\tau}, \\
& e^{-p_{\perp}^{2} /\left|e B_{0, \lambda}\right|}\left[v_{F}\left(\nabla_{3} T\right) \partial_{T}+\left(-s_{B} \lambda\right) e E_{3} \partial_{p_{3}}\right] \tilde{f}_{L L L}\left(p_{3}\right)=-\frac{w_{3}+s_{B} \lambda e^{-p_{\perp}^{2} /\left|e B_{0, \lambda}\right|} \tilde{f}_{\mathrm{LLL}}\left(p_{3}\right)}{\tau}
\end{aligned}
$$

where $\tau$ is the relaxation time. As is easy to check, $w_{1}=w_{2}=0$ in the present setup. It is worth noting that, in realistic models, the relaxation time may depend on the particle energy, external fields, as well as other parameters. For simplicity, here we will assume that $\tau$ is a constant. The solutions to eqs. (6.2a) and (6.2b) read

$$
\begin{aligned}
& w_{0}=e^{-p_{\perp}^{2} /\left|e B_{0, \lambda}\right|}\left\{\tilde{f}_{L L L}\left(p_{3}\right)-\tau\left[\left(-s_{B} \lambda\right) v_{F} \nabla_{3} T \partial_{T} \tilde{f}_{L L L}\left(p_{3}\right)+e E_{3} \partial_{p_{3}} \tilde{f}_{L L L}\left(p_{3}\right)\right]\right\}, \\
& w_{3}=-s_{B} \lambda e^{-p_{\perp}^{2} /\left|e B_{0, \lambda}\right|}\left\{\tilde{f}_{L L L}\left(p_{3}\right)-\tau\left[\left(-s_{B} \lambda\right) v_{F} \nabla_{3} T \partial_{T} \tilde{f}_{L L L}\left(p_{3}\right)+e E_{3} \partial_{p_{3}} \tilde{f}_{L L L}\left(p_{3}\right)\right]\right\} .
\end{aligned}
$$

By making use of these results, we can now calculate the electric and heat current densities. In terms of the Wigner function, the corresponding current densities are given by (see, e.g., ref. [47])

$$
\begin{aligned}
& j_{3}=-e v_{F} \sum_{\lambda= \pm} \lambda \int \frac{d^{3} \mathbf{p}}{(2 \pi)^{3}} \operatorname{tr}\left[\sigma_{3} W\right]=-2 e v_{F} \sum_{\lambda= \pm} \int \frac{d^{3} \mathbf{p}}{(2 \pi)^{3}} w_{3} \\
& j_{3}^{Q}=v_{F} \sum_{\lambda= \pm} \lambda \int \frac{d^{3} \mathbf{p}}{(2 \pi)^{3}}\left(\epsilon_{0}-\mu_{\lambda}\right) \operatorname{tr}\left[\sigma_{3} W\right]=2 v_{F} \sum_{\lambda= \pm} \int \frac{d^{3} \mathbf{p}}{(2 \pi)^{3}}\left(s_{B} \lambda v_{F} p_{3}-\mu_{\lambda}\right) w_{3}
\end{aligned}
$$

The calculation reduces to four types of integrals, presented in eqs. (D.6) through (D.9) in appendix D. By making use of the corresponding results, we arrive at the following final 
expressions for the current densities:

$$
\begin{aligned}
j_{3} & =\sum_{\lambda= \pm} 2 e v_{F} s_{B} \lambda\left\{I_{1}-\tau\left[\left(-s_{B} \lambda\right) v_{F}\left(\nabla_{3} T\right)\left(\partial_{T} I_{1}\right)+e E_{3} I_{3}\right]\right\} \\
& =\sum_{\lambda= \pm} \frac{\lambda e^{2} B_{0, \lambda} \mu_{\lambda}}{(2 \pi)^{2}}+\tau \sum_{\lambda= \pm} \frac{\left|e B_{0, \lambda}\right| e^{2} E_{3} v_{F}}{(2 \pi)^{2}}, \\
j_{3}^{Q} & =-\sum_{\lambda= \pm} 2 v_{F} s_{B} \lambda\left\{I_{2}-\mu_{\lambda} I_{1}-\tau\left[\left(-s_{B} \lambda\right) v_{F}\left(\nabla_{3} T\right) \partial_{T}\left(I_{2}-\mu_{\lambda} I_{1}\right)+e E_{3}\left(I_{4}-\mu_{\lambda} I_{3}\right)\right]\right\} \\
& =\sum_{\lambda= \pm} \frac{\lambda e B_{0, \lambda}}{2(2 \pi)^{2}}\left[\mu_{\lambda}^{2}-\frac{\pi^{2} T^{2}}{3}+v_{F}^{2} \Lambda^{2}\right]-\tau v_{F}\left(\nabla_{3} T\right) \sum_{\lambda= \pm} \frac{\left|e B_{0, \lambda}\right| T}{12} .
\end{aligned}
$$

The last term in eq. (6.5a) is similar to the usual Drude conductivity and is related to the density of states on the LLL. Similar physical interpretation applies to the last term in eq. (6.5b) connected with the heat transport. Note that the anomalous Nernst effect, i.e., the correction to $\mathbf{j}$ proportional to the cross product of the Berry curvature and the temperature gradient [45], is absent in the problem at hand. This fact is not surprising in view of the one-dimensional nature of the LLL. Last but not least, we note that the last term in the square brackets in eq. (6.5b) diverges. The corresponding contribution should be naturally regularized in realistic lattice models.

By comparing eqs. (6.5a) and (6.5b) with the general linear response relation, i.e.,

$$
\begin{aligned}
& j_{3}=\tilde{j}_{3}+\sigma_{33}^{(e e)} E_{3}+\sigma_{33}^{(e T)}\left(-\nabla_{3} T\right), \\
& j_{3}^{Q}=\tilde{j}_{3}^{Q}+\sigma_{33}^{(T e)} E_{3}+\sigma_{33}^{(T T)}\left(-\nabla_{3} T\right),
\end{aligned}
$$

where $\tilde{j}_{3}$ and $\tilde{j}_{3}^{Q}$ denote the nondissipative parts of the currents proportional to $e B_{0}$ and $e B_{0,5}$ that come from the first terms in eqs. (6.5a) and (6.5b) [see also eqs. (3.13) and (3.14)], we find that the off-diagonal terms of the thermoelectric conductivity tensor vanish, i.e., $\sigma_{33}^{(e T)}=T \sigma_{33}^{(T e)}=0$. Therefore, the Seebeck coefficient (or thermopower) $S=\sigma_{33}^{(e T)} / \sigma_{33}^{(e e)}$ vanishes too. The thermoconductivity is defined in the absence of electric current and equals to

$$
\kappa_{33}=\sigma_{33}^{(T T)}-\frac{\sigma_{33}^{(T e)} \sigma_{33}^{(e T)}}{\sigma_{33}^{(e e)}}=\sigma_{33}^{(T T)} .
$$

Finally, one can easily check that the Wiedemann-Franz law $\kappa_{33}=T \sigma_{33}^{(e e)} \pi^{2} /\left(3 e^{2}\right)$ and the Mott relation $\sigma_{33}^{(e T)}=\pi^{2} T\left(\partial_{\mu} \sigma_{33}^{(e e)}\right) /(3 e)=0$ are satisfied. In fact, in the present setup, $\kappa_{33}$ and $\sigma_{33}^{(e e)}$ are the only nonzero components of the thermal and electric conductivity tensors, respectively.

In the case of the vanishing axial magnetic field, $B_{0,5}=0$, eqs. (6.5a) and (6.5b) take the following simpler form:

$$
\begin{aligned}
j_{3} & =\frac{e^{2} B_{0} \mu_{5}}{2 \pi^{2}}+\tau \frac{e^{2} E_{3} v_{F}\left|e B_{0}\right|}{2 \pi^{2}}, \\
j_{3}^{Q} & =\frac{e B_{0} \mu \mu_{5}}{2 \pi^{2}}-\tau v_{F}\left|e B_{0}\right| \frac{T \nabla_{3} T}{6} .
\end{aligned}
$$


On the other hand, when the ordinary magnetic field vanishes, but a nonzero axial magnetic field is present, the electric current density is given by

$$
j_{3}=\frac{e^{2} B_{0,5} \mu}{2 \pi^{2}}+\tau \frac{e^{2} E_{3} v_{F}\left|e B_{0,5}\right|}{2 \pi^{2}} .
$$

As we see, this is similar to that in eq. (6.8a). However, this is not the case for the heat current density, i.e.,

$$
j_{3}^{Q}=\frac{e B_{0,5}}{(2 \pi)^{2}}\left(\mu^{2}+\mu_{5}^{2}-\frac{\pi^{2} T^{2}}{3}+v_{F}^{2} \Lambda^{2}\right)-\tau v_{F}\left|e B_{0,5}\right| \frac{T \nabla_{3} T}{6} .
$$

While the dissipative term proportional to the relaxation time is similar to the corresponding term in eq. (6.8b), the nondissipative ones are completely different. For example, they do not require the presence of both chemical and chiral chemical potentials and could be nonzero even at $\mu=\mu_{5}=0$.

Before concluding this section, it is instructive to point that all nondissipative contributions in eqs. (6.9) and (6.10), as well as in eqs. (6.8a) and (6.8b), are bound currents that can be expressed as curls of other quantities. (In other words, their structure is similar to the magnetization current $\mathbf{j}_{M} \sim[\boldsymbol{\nabla} \times \mathbf{M}]$.) This follows from the fact that both $\mathbf{B}_{0}$ and $\mathbf{B}_{0,5}$ can be expressed as curls of the vector $\mathbf{A}$ and axial vector $\mathbf{A}_{5}$ potentials, respectively. It is interesting to note here that, in the context of Weyl semimetals, the axial potential $\mathbf{A}_{5}$ (unlike the usual vector potential $\mathbf{A}$ ) is an observable quantity [54-58] which is related to the separation between Weyl nodes in the momentum space.

\section{Summary}

By using the exact solutions of the Weyl equation for chiral fermions in constant magnetic and axial magnetic fields, we calculated the equal-time Wigner function for a magnetized chiral fermion plasma at finite chemical potential and temperature. This exact Wigner function is defined by the scalar and vector parts in the basis of the Pauli matrices. While the vector part is necessary to calculate currents, the scalar part defines the Wigner quasiprobability distribution function. We checked that, to the linear order in magnetic field, the latter also agrees with the distribution functions of the chiral kinetic theory. It is interesting to note that, owing to its quantum nature, the scalar part of the Wigner function can be negative in a magnetic field. Besides the possibility of negative values (assuming $\mu_{\lambda}>0$ ), the most crucial difference between the standard quasiprobability function, which is given in terms of the Fermi-Dirac functions, and the Wigner quasiprobability distribution function in a magnetic field is connected with the chirality-correlated asymmetric dependence of the latter on the longitudinal component of momentum. A similar asymmetric dependence is found in the vector part of the Wigner function and is principal for reproducing the correct chiral magnetic and chiral separation effects.

Retaining only the lowest Landau level contribution, the equation for the equal-time Wigner function in a strong magnetic field is obtained. The constant background magnetic and axial magnetic (or, equivalently, strain-induced pseudomagnetic) fields are taken into 
account nonperturbatively. In this case the scalar and vector parts of the Wigner function are both proportional to the distribution function on the LLL. By making use of this equation, it is found that the longitudinal collective excitations in a strong magnetic field are gapped plasmons. The magnitude of their energy gap is determined by the value of the magnetic field. Interestingly, the situation changes qualitatively in the case of the axial magnetic field. The dispersion relation of the corresponding collective excitation, identified as the chiral pseudomagnetic wave, is clearly asymmetric in the wave vector. While the chiral pseudomagnetic wave appears to be gapless in the LLL approximation, we argued that the corresponding mode is in fact gapped when higher Landau levels are included. As in the limit of a weak axial magnetic field $[69,70]$, the minimum energy of the corresponding mode should be at $k_{3} \sim B_{0,5}$.

By making use of the Wigner function in the LLL approximation, we also studied the thermoelectric transport of chiral fermions in a strong magnetic field. The analysis was performed in a phenomenological model where the effects of collisions were introduced into the equation for the Wigner function via a constant relaxation time. The latter, of course, is not a very realistic approximation to capture all details of the thermoelectric transport, but should be sufficient at least for understanding qualitative features. We found that the electric and heat (thermal) current densities are determined by the density of states on the lowest Landau level. While the nondissipative part of the heat current density in a magnetic field requires the presence of both chemical and chiral chemical potentials, its counterpart in an axial magnetic field is nontrivial when at least one of these parameters or temperature is present. All nondissipative contributions to currents come in the form of bound currents that are curls of other quantities. The structures of the dissipative terms are similar (up to the interchange $B_{0,5} \leftrightarrow B_{0}$ ) in the cases of background magnetic $B_{0}$ and axial magnetic $B_{0,5}$ fields.

\section{Acknowledgments}

The work of E.V.G. was supported partially by the Ukrainian State Foundation for Fundamental Research. The work of V.A.M. and P.O.S. was supported by the Natural Sciences and Engineering Research Council of Canada. The work of I.A.S. was supported in part by the U.S. National Science Foundation under Grant No. PHY-1404232.

Note added. When finishing this paper, we became aware of a partially overlapping study by Xin-li Sheng, Dirk H. Rischke, David Vasak, and Qun Wang [73].

\section{A Wave functions of the Weyl Hamiltonian}

In this appendix, we derive the wave functions of the model Hamiltonian (2.1) for the Weyl fermions in a constant magnetic field. In order to solve the eigenvalue problem $H_{\lambda} \psi_{\lambda}=E \psi_{\lambda}$, we look for a solution in the form $\psi_{\lambda}(\mathbf{x})=e^{i p_{3} x_{3}+i p_{2} x_{2}-i E t} \varphi_{\lambda}(\xi)$, where the new variable $\xi$ is defined by

$$
\xi=\sqrt{\left|e B_{\lambda}\right|}\left(\frac{p_{2}}{e B_{\lambda}}+x_{1}\right)
$$


By noting that $\partial_{x_{1}}=\partial_{\xi} \sqrt{\left|e B_{\lambda}\right|}$, we check that function $\varphi_{\lambda}$ satisfies the following ordinary differential equation:

$$
\left[\partial_{\xi}-s_{B} \sigma_{z} \xi-\frac{i \lambda \sigma_{x}}{v_{F} \sqrt{\left|e B_{\lambda}\right|}}\left(E-\mu_{\lambda}-\lambda v_{F} p_{3} \sigma_{z}\right)\right] \varphi_{\lambda}=0
$$

where $s_{B}=\operatorname{sgn}\left(e B_{\lambda}\right)$. The solution to this equation can be given in the form

$$
\varphi_{\lambda}=\Phi_{\lambda}^{+}(\xi) u_{\lambda}^{+}+\Phi_{\lambda}^{-}(\xi) u_{\lambda}^{-},
$$

where $u_{\lambda}^{ \pm}$are two linearly independent spinors that satisfy the following relations:

$$
\sigma_{z} u_{\lambda}^{ \pm}= \pm u_{\lambda}^{ \pm}, \quad u_{\lambda}^{\mp}=\frac{\lambda \sigma_{x}\left(E-\mu_{\lambda}-\lambda v_{F} p_{3} \sigma_{z}\right)}{\sqrt{\left(E-\mu_{\lambda}\right)^{2}-v_{F}^{2} p_{3}^{2}}} u_{\lambda}^{ \pm} .
$$

After substituting the ansatz (A.3) into eq. (A.2) and separating linearly independent terms proportional to spinors $u_{\lambda}^{ \pm}$, we arrive at the following coupled set of equations:

$$
\begin{array}{r}
{\left[\partial_{\xi} \Phi_{\lambda}^{ \pm}(\xi) \mp s_{B} \xi \Phi_{\lambda}^{ \pm}(\xi)-i k \Phi_{\lambda}^{\mp}(\xi)\right]=0} \\
{\left[\partial_{\xi}^{2} \mp s_{B}-\xi^{2}+k^{2}\right] \Phi_{\lambda}^{ \pm}(\xi)=0}
\end{array}
$$

where

$$
k \equiv \sqrt{\frac{\left(E-\mu_{\lambda}\right)^{2}-v_{F}^{2} p_{3}^{2}}{v_{F}^{2}\left|e B_{\lambda}\right|}} .
$$

Solutions of eqs. (A.5) and (A.6) can be expressed in terms of the parabolic cylinder functions $D_{p}(\alpha \xi)[74,75]$, i.e.,

$$
\Phi_{\lambda}^{-s_{B}}(\xi)=D_{k^{2} / 2}(\sqrt{2} \xi), \quad \Phi_{\lambda}^{s_{B}}(\xi)=-\frac{i k}{\sqrt{2}} D_{k^{2} / 2-1}(\sqrt{2} \xi) .
$$

By making use of eq. (A.4), we also determine the explicit form of spinors $u_{\lambda}^{ \pm}$for each of the two possible choices of $s_{B}= \pm 1$. The result reads

$$
\begin{aligned}
u_{\lambda}^{s_{B}} & =\mathcal{P}_{s_{B}}\left(\begin{array}{c}
\lambda \frac{E-\mu_{\lambda}+\lambda s_{B} v_{F} p_{3}}{v_{F} \sqrt{2 n\left|e B_{\lambda}\right|}} \\
0
\end{array}\right), \\
u_{\lambda}^{-s_{B}} & =\mathcal{P}_{s_{B}}\left(\begin{array}{l}
0 \\
1
\end{array}\right),
\end{aligned}
$$

where

$$
\mathcal{P}_{s_{B}}=\frac{\left(1-s_{B}\right)}{2} \sigma_{x}+\frac{\left(1+s_{B}\right)}{2}
$$

is a matrix operator that interchanges the two components of the spinor when the sign $s_{B}=\operatorname{sgn}\left(e B_{\lambda}\right)$ changes.

The requirement of finite wave functions at $|\xi| \rightarrow \infty$ leads to the constraint $k^{2} / 2=n$, where $n=0,1,2 \ldots$. Then, the parabolic cylinder functions can be expressed in terms of the Hermitian polynomials [74, 75]. After fixing the overall normalization constants (by 
using formula 7.374.1 in ref. [75]), we finally obtain the following eigenfunctions of the Weyl Hamiltonian (2.1):

$$
\begin{aligned}
\psi_{n=0, p_{2}, p_{3}=} & \left|e B_{\lambda}\right|^{1 / 4} e^{i p_{3} x_{3}+i p_{2} x_{2}-i E t} Y_{0}(\xi) \mathcal{P}_{s_{B}}\left(\begin{array}{l}
0 \\
1
\end{array}\right), \\
\psi_{n>0, p_{2}, p_{3}=} & \left|e B_{\lambda}\right|^{1 / 4} \sqrt{\frac{2 v_{F}^{2} n\left|e B_{\lambda}\right|}{2 v_{F}^{2} n\left|e B_{\lambda}\right|+\left[E_{n}-\mu_{\lambda}+s_{B} v_{F} \lambda p_{z}\right]^{2}}} e^{i p_{3} x_{3}+i p_{2} x_{2}-i E t} \mathcal{P}_{s_{B}} \\
& \times\left\{Y_{n}(\xi)\left(\begin{array}{l}
0 \\
1
\end{array}\right)-i \lambda \frac{\left(E_{n}-\mu_{\lambda}+s_{B} v_{F} \lambda p_{z}\right)}{v_{F} \sqrt{2 n\left|e B_{\lambda}\right|}} Y_{n-1}(\xi)\left(\begin{array}{l}
1 \\
0
\end{array}\right)\right\},
\end{aligned}
$$

where

$$
Y_{n}(\xi)=\frac{1}{\sqrt{2^{n} n ! \sqrt{\pi}}} e^{-\xi^{2} / 2} H_{n}(\xi)
$$

The corresponding energies for the lowest $(n=0)$ and higher $(n>0)$ Landau levels are

$$
\begin{aligned}
& E_{n=0}=\mu_{\lambda}-s_{B} v_{F} \lambda p_{3}=\mu_{\lambda}+\epsilon_{0}, \\
& E_{n>0}=\mu_{\lambda} \pm v_{F} \sqrt{p_{3}^{2}+2 n\left|e B_{\lambda}\right|}=\mu_{\lambda}+\epsilon_{n>0},
\end{aligned}
$$

respectively. [Note that in the main text we changed the sign at $p_{3}$ in order to use the same notations in the Wigner function, where momenta are opposite with respect to that in the wave functions.]

\section{B Derivation of the Wigner function in a constant magnetic field}

In this appendix, we provide the details of the calculation of the equal-time Wigner function of chiral fermions in a constant external magnetic field. Let us write the Wigner operator (3.1) explicitly

$$
\begin{aligned}
\hat{W}_{\alpha \eta}(\mathbf{x}, \mathbf{p})=\frac{1}{2} \sum_{n, n^{\prime}} \int d^{3} \mathbf{y} \int \frac{d^{2} \mathbf{q}}{(2 \pi)^{2}} \int \frac{d^{2} \mathbf{q}^{\prime}}{(2 \pi)^{2}} e^{-i \mathbf{p} \cdot \mathbf{y}} e^{i \Phi\left(\mathbf{r}_{+}, \mathbf{r}_{-}\right)} \\
\times\left\{\left[2 \hat{a}_{n, \mathbf{q}}^{\dagger} \hat{a}_{n^{\prime}, \mathbf{q}^{\prime}}-(2 \pi)^{2} \delta_{n, n^{\prime}} \delta\left(\mathbf{q}-\mathbf{q}^{\prime}\right)\right] \psi_{\eta, n, \mathbf{q}}^{\dagger}\left(\mathbf{r}_{+}\right) \psi_{\alpha, n^{\prime}, \mathbf{q}^{\prime}}\left(\mathbf{r}_{-}\right)\right. \\
+\left[\hat{a}_{n, \mathbf{q}}^{\dagger} \hat{b}_{n^{\prime}, \mathbf{q}^{\prime}}^{\dagger}-\hat{b}_{n^{\prime}, \mathbf{q}^{\prime}}^{\dagger} \hat{a}_{n, \mathbf{q}}^{\dagger}\right] \psi_{\eta, n, \mathbf{q}}^{\dagger}\left(\mathbf{r}_{+}\right) \phi_{\alpha, n^{\prime}, \mathbf{q}^{\prime}}\left(\mathbf{r}_{-}\right) \\
+\left[\hat{b}_{n, \mathbf{q}} \hat{a}_{n^{\prime}, \mathbf{q}^{\prime}}-\hat{a}_{n^{\prime}, \mathbf{q}^{\prime}} \hat{b}_{n, \mathbf{q}}\right] \phi_{\eta, n, \mathbf{q}}^{\dagger}\left(\mathbf{r}_{+}\right) \psi_{\alpha, n^{\prime}, \mathbf{q}^{\prime}}\left(\mathbf{r}_{-}\right) \\
\left.-\left[2 \hat{b}_{n, \mathbf{q}^{\dagger}}^{\dagger} \hat{b}_{n^{\prime}, \mathbf{q}^{\prime}}-(2 \pi)^{2} \delta_{n, n^{\prime}} \delta\left(\mathbf{q}-\mathbf{q}^{\prime}\right)\right] \phi_{\eta, n, \mathbf{q}}^{\dagger}\left(\mathbf{r}_{+}\right) \phi_{\alpha, n^{\prime}, \mathbf{q}^{\prime}}\left(\mathbf{r}_{-}\right)\right\}
\end{aligned}
$$

where $\mathbf{r}_{ \pm}=\mathbf{x} \pm \mathbf{y} / 2$, the phase $\Phi\left(\mathbf{r}_{+}, \mathbf{r}_{-}\right)=-e \int_{\mathbf{r}_{-}}^{\mathbf{r}_{+}} d \mathbf{r} \mathbf{A}_{\lambda}(\mathbf{r})=-e B_{\lambda} y_{2} x_{1}$ ensures the gauge invariance of $\hat{W}_{\alpha \eta}$, and we used the standard anticommutation relations for the fermion particle creation and annihilation operators $\hat{a}_{n, \mathbf{q}}^{\dagger}, \hat{a}_{n, \mathbf{q}}$, as well as their antiparticle $\hat{b}_{n, \mathbf{q}}^{\dagger}, \hat{b}_{n, \mathbf{q}}$ counterparts. While the spinors for particle states $\psi_{n, \mathbf{q}}$ are given by eqs. (A.12) and (A.13), 
the spinors for antiparticles are defined by $\left.\phi_{n, \mathbf{q}} \equiv \psi_{n, \mathbf{q}}\right|_{\epsilon_{n} \rightarrow-\mid \epsilon_{n}}$. For simplicity, we set also $\mathbf{q}=\left(q_{2}, q_{3}\right)$ and $\mathbf{q}^{\prime}=\left(q_{2}^{\prime}, q_{3}^{\prime}\right)$.

The Wigner function is defined as an average of the Wigner operator over the Hilbert space of the multi-particle states $|\Phi\rangle=\left|\ldots, N_{m_{i}}, \ldots, \ldots, \bar{N}_{m_{i}}, \ldots\right\rangle$ with $N_{m_{i}}$ particles in state $m_{i}$, and $\bar{N}_{m_{i}}$ antiparticles in state $m_{i}$, i.e.,

$$
\begin{aligned}
W_{\alpha \eta}(\mathbf{x}, \mathbf{p})= & \operatorname{Tr}\left(\hat{W}_{\alpha \eta}(\mathbf{x}, \mathbf{p}) \hat{\rho}\right)=\sum_{\Phi}\langle\Phi|\hat{W}(\mathbf{x}, \mathbf{p}) \hat{\rho}| \Phi\rangle \\
= & \sum_{n=0}^{\infty} \int d^{3} \mathbf{y} \int \frac{d^{2} \mathbf{q}}{(2 \pi)^{2}} e^{-i \mathbf{p} \cdot \mathbf{y}-i e B_{\lambda} y_{2} x_{1}} \sum_{\epsilon_{n}} \operatorname{tr}\left\{\psi_{\eta, n, \mathbf{q}}^{\dagger}\left(\mathbf{r}_{+}\right) \psi_{\alpha, n, \mathbf{q}}\left(\mathbf{r}_{-}\right) \frac{\theta\left(\epsilon_{n}(\mathbf{q})\right)}{1+e^{\beta\left(\epsilon_{n}(\mathbf{q})-\mu_{\lambda}\right)}}\right. \\
& -\phi_{\eta, n, \mathbf{q}}^{\dagger}\left(\mathbf{r}_{+}\right) \phi_{\alpha, n, \mathbf{q}}\left(\mathbf{r}_{-}\right) \frac{\theta\left(-\epsilon_{n}(\mathbf{q})\right)}{1+e^{-\beta\left(\epsilon_{n}(\mathbf{q})-\mu_{\lambda}\right)}} \\
& \left.-\frac{1}{2}\left[\theta\left(\epsilon_{n}(\mathbf{k})\right) \psi_{\eta, n, \mathbf{q}}^{\dagger}\left(\mathbf{r}_{+}\right) \psi_{\alpha, n, \mathbf{q}}\left(\mathbf{r}_{-}\right)-\theta\left(-\epsilon_{n}(\mathbf{k})\right) \phi_{\eta, n, \mathbf{q}}^{\dagger}\left(\mathbf{r}_{+}\right) \phi_{\alpha, n, \mathbf{q}}\left(\mathbf{r}_{-}\right)\right]\right\} .
\end{aligned}
$$

Here the density matrix operator $\hat{\rho}$ is given by eq. (3.3) in the main text. Further, $\beta=1 / T$ is the inverse temperature, $\sum_{\epsilon_{n}}$ denotes the summation over positive and negative branches of the energy spectrum, and $\theta$-functions are the unit step functions which select the proper sign of the energy of particles and antiparticles.

In order to proceed with the evaluation of the Wigner function (B.2), we first calculate

$$
\begin{aligned}
& \int d^{3} \mathbf{y} e^{-i \mathbf{p} \cdot \mathbf{y}} e^{-i e B_{\lambda} y_{2} x_{1}} \psi_{\eta, n, \mathbf{q}}^{\dagger} \psi_{\alpha, n, \mathbf{q}}=\int d^{3} \mathbf{y} e^{-i \mathbf{p} \cdot \mathbf{y}} e^{-i e B_{\lambda} y_{2} x_{1}-i q_{3} y_{3}-i q_{2} y_{2}} \\
& \times \frac{\sqrt{\left|e B_{\lambda}\right|}}{1+\frac{\left[\epsilon_{n}\left(q_{3}\right)+s_{B} v_{F} \lambda q_{3}\right]^{2}}{2 v_{F}^{2} n\left|e B_{\lambda}\right|}}\left\{Y_{n}\left(\xi_{+}\right) Y_{n}\left(\xi_{-}\right)\left[\frac{1+s_{B}}{2} \delta_{\alpha 2} \delta_{\eta 2}+\frac{1-s_{B}}{2} \delta_{\alpha 1} \delta_{\eta 1}\right]\right. \\
& +\frac{\left[\epsilon_{n}\left(q_{3}\right)+s_{B} v_{F} \lambda q_{3}\right]^{2}}{2 n v_{F}^{2}\left|e B_{\lambda}\right|} Y_{n-1}\left(\xi_{+}\right) Y_{n-1}\left(\xi_{-}\right)\left[\frac{1-s_{B}}{2} \delta_{\alpha 2} \delta_{\eta 2}+\frac{1+s_{B}}{2} \delta_{\alpha 1} \delta_{\eta 1}\right] \\
& -Y_{n}\left(\xi_{+}\right) Y_{n-1}\left(\xi_{-}\right) \frac{i \lambda\left[\epsilon_{n}\left(q_{3}\right)+s_{B} \lambda v_{F} q_{3}\right]}{v_{F} \sqrt{2 n\left|e B_{\lambda}\right|}}\left[\frac{1-s_{B}}{2} \delta_{\alpha 2} \delta_{\eta 1}+\frac{1+s_{B}}{2} \delta_{\alpha 1} \delta_{\eta 2}\right] \\
& \left.+Y_{n-1}\left(\xi_{+}\right) Y_{n}\left(\xi_{-}\right) \frac{i \lambda\left[\epsilon_{n}\left(q_{3}\right)+s_{B} \lambda v_{F} q_{3}\right]}{v_{F} \sqrt{2 n\left|e B_{\lambda}\right|}}\left[\frac{1-s_{B}}{2} \delta_{\alpha 1} \delta_{\eta 2}+\frac{1+s_{B}}{2} \delta_{\alpha 2} \delta_{\eta 1}\right]\right\},
\end{aligned}
$$

where

$$
\xi_{ \pm}=\sqrt{\left|e B_{\lambda}\right|}\left(\frac{q_{2}}{e B_{\lambda}}+x_{1} \pm \frac{y_{1}}{2}\right), \quad \xi=\sqrt{\left|e B_{\lambda}\right|}\left(\frac{q_{2}}{e B_{\lambda}}+x_{1}\right)
$$

Using the table integral 7.377 in ref. [75], we find the following auxiliary expressions:

$$
\begin{aligned}
& \int d y_{1} e^{-i p_{1} y_{1}} e^{-\frac{\left|e B_{\lambda}\right| y_{1}^{2}}{4}} H_{n}\left[\sqrt{\left|e B_{\lambda}\right|}\left(\frac{q_{2}}{e B_{\lambda}}+x_{1}+\frac{y_{1}}{2}\right)\right] H_{n}\left[\sqrt{\left|e B_{\lambda}\right|}\left(\frac{q_{2}}{e B_{\lambda}}+x_{1}-\frac{y_{1}}{2}\right)\right] \\
& =(-1)^{n} \frac{e^{-p_{1}^{2} /\left|e B_{\lambda}\right|}}{\sqrt{\left|e B_{\lambda}\right|}} 2^{n+1} \sqrt{\pi} n ! L_{n}\left[2\left(\xi^{2}+\frac{p_{1}^{2}}{\left|e B_{\lambda}\right|}\right)\right]
\end{aligned}
$$


and

$$
\begin{gathered}
\int d y_{1} e^{-i p_{1} y_{1}} e^{-\frac{\left|e B_{\lambda}\right| y_{1}^{2}}{4}} H_{n}\left[\sqrt{\left|e B_{\lambda}\right|}\left(\frac{q_{2}}{e B_{\lambda}}+x_{1} \pm \frac{y_{1}}{2}\right)\right] H_{n-1}\left[\sqrt{\left|e B_{\lambda}\right|}\left(\frac{q_{2}}{e B_{\lambda}}+x_{1} \mp \frac{y_{1}}{2}\right)\right] \\
=(-1)^{n-1} \frac{e^{-p_{1}^{2} /\left|e B_{\lambda}\right|}}{\sqrt{\left|e B_{\lambda}\right|}} 2^{n+1} \sqrt{\pi}(n-1) !\left(\xi-\frac{i p_{1}}{\sqrt{\left|e B_{\lambda}\right|}}\right) L_{n-1}^{1}\left[2\left(\xi^{2}+\frac{p_{1}^{2}}{\left|e B_{\lambda}\right|}\right)\right], \quad \text { B.6 }
\end{gathered}
$$

where $L_{n}^{m}(x)$ are the generalized Laguerre polynomials [75]. The above expressions allows us to obtain the diagonal

$$
\begin{aligned}
\int & d^{3} \mathbf{y} e^{-i \mathbf{p} \cdot \mathbf{y}} e^{-i e B_{\lambda} y_{2} x_{1}} \psi_{\alpha, n, \mathbf{q}}^{\dagger} \psi_{\alpha, n, \mathbf{q}}=\frac{1}{2 \pi} \delta\left(p_{3}+q_{3}\right) \delta\left(p_{2}+q_{2}+e B_{\lambda} x_{1}\right) e^{-\xi^{2}-p_{1}^{2} /\left|e B_{\lambda}\right|} \\
& \times \frac{4(-1)^{n} v_{F}^{2} n\left|e B_{\lambda}\right|}{2 v_{F}^{2} n\left|e B_{\lambda}\right|+\left[\epsilon_{n}\left(q_{3}\right)+s_{B} v_{F} \lambda q_{3}\right]^{2}}\left\{L_{n}\left[2\left(\xi^{2}+\frac{p_{1}^{2}}{\left|e B_{\lambda}\right|}\right)\right]\left[\frac{1+s_{B}}{2} \delta_{\alpha 2}+\frac{1-s_{B}}{2} \delta_{\alpha 1}\right]\right. \\
& \left.-\frac{\left[\epsilon_{n}\left(q_{3}\right)+s_{B} v_{F} \lambda q_{3}\right]^{2}}{2 v_{F}^{2} n\left|e B_{\lambda}\right|} L_{n-1}\left[2\left(\xi^{2}+\frac{p_{1}^{2}}{\left|e B_{\lambda}\right|}\right)\right]\left[\frac{1-s_{B}}{2} \delta_{\alpha 2}+\frac{1+s_{B}}{2} \delta_{\alpha 1}\right]\right\}
\end{aligned}
$$

and off-diagonal $\alpha \neq \eta$

$$
\begin{aligned}
\int & d^{3} \mathbf{y} e^{-i \mathbf{p} \cdot \mathbf{y}} e^{-i e B_{\lambda} y_{2} x_{1}} \psi_{\eta, n, \mathbf{q}}^{\dagger} \psi_{\alpha, n, \mathbf{q}}=\frac{1}{2 \pi} \delta\left(p_{3}+q_{3}\right) \delta\left(p_{2}+q_{2}+e B_{\lambda} x_{1}\right) \frac{-i \lambda\left[\epsilon_{n}\left(q_{3}\right)+s_{B} \lambda v_{F} q_{3}\right]}{v_{F} n \sqrt{\left|e B_{\lambda}\right|}} \\
& \times \frac{4(-1)^{n-1} v_{F}^{2} n\left|e B_{\lambda}\right| e^{-\xi^{2}-p_{1}^{2} /\left|e B_{\lambda}\right|}}{2 v_{F}^{2} n\left|e B_{\lambda}\right|+\left[\epsilon_{n}\left(q_{3}\right)+s_{B} v_{F} \lambda q_{3}\right]^{2}} L_{n-1}^{1}\left[2\left(\xi^{2}+\frac{p_{1}^{2}}{\left|e B_{\lambda}\right|}\right)\right] \\
& \times\left\{\left(\frac{s_{B} q_{2}-i p_{1}}{\sqrt{\left|e B_{\lambda}\right|}}+\sqrt{\left|e B_{\lambda}\right|} x_{1}\right)\left[\frac{1+s_{B}}{2} \delta_{\alpha 1} \delta_{\eta 2}+\frac{1-s_{B}}{2} \delta_{\alpha 2} \delta_{\eta 1}\right]\right. \\
& \left.-\left(\frac{s_{B} q_{2}+i p_{1}}{\sqrt{\left|e B_{\lambda}\right|}}+\sqrt{\left|e B_{\lambda}\right|} x_{1}\right)\left[\frac{1-s_{B}}{2} \delta_{\alpha 1} \delta_{\eta 2}+\frac{1+s_{B}}{2} \delta_{\alpha 2} \delta_{\eta 1}\right]\right\},
\end{aligned}
$$

parts of the Wigner function $W_{\alpha \eta}(\mathbf{x}, \mathbf{p})$. The latter can be represented in the following matrix form:

$$
W(\mathbf{x}, \mathbf{p})=\mathcal{P}_{s_{B}}\left(\begin{array}{ll}
W_{11} & W_{12} \\
W_{21} & W_{22}
\end{array}\right) \mathcal{P}_{s_{B}}^{-1},
$$

where

$$
\begin{aligned}
W_{11}= & -e^{-p_{\perp}^{2} /\left|e B_{\lambda}\right|} \sum_{n=0}^{\infty} \sum_{\epsilon_{n}} \frac{(-1)^{n} L_{n-1}\left(\frac{2 p_{\perp}^{2}}{\left|e B_{\lambda}\right|}\right)}{\left|\epsilon_{n}\right|}\left\{\left(\left|\epsilon_{n}\right|-s_{B} \lambda v_{F} p_{3}\right) \frac{\theta\left(\epsilon_{n}\right)}{1+e^{\beta\left(\left|\epsilon_{n}\right|-\mu_{\lambda}\right)}}\right. \\
& \left.-\left(\left|\epsilon_{n}\right|+s_{B} \lambda v_{F} p_{3}\right) \frac{\theta\left(-\epsilon_{n}\right)}{1+e^{\beta\left(\left|\epsilon_{n}\right|+\mu_{\lambda}\right)}}-\frac{1}{2}\left[\epsilon_{n}-s_{B} \lambda v_{F} p_{3}\right]\right\} \\
W_{22}= & e^{-p_{\perp}^{2} /\left|e B_{\lambda}\right|} \sum_{n=0}^{\infty} \sum_{\epsilon_{n}} \frac{(-1)^{n} L_{n}\left(\frac{2 p_{\perp}^{2}}{\left|e B_{\lambda}\right|}\right)}{\left|\epsilon_{n}\right|}\left\{\left(\left|\epsilon_{n}\right|+s_{B} v_{F} \lambda p_{3}\right) \frac{\theta\left(\epsilon_{n}\right)}{1+e^{\beta\left(\left|\epsilon_{n}\right|-\mu_{\lambda}\right)}}\right. \\
& \left.-\left(\left|\epsilon_{n}\right|-s_{B} v_{F} \lambda p_{3}\right) \frac{\theta\left(-\epsilon_{n}\right)}{1+e^{\beta\left(\left|\epsilon_{n}\right|+\mu_{\lambda}\right)}}-\frac{1}{2}\left[\epsilon_{n}+s_{B} \lambda v_{F} p_{3}\right]\right\}
\end{aligned}
$$




$$
\begin{aligned}
W_{12}= & W_{21}^{*}=2 e^{-p_{\perp}^{2} /\left|e B_{\lambda}\right|} \sum_{n=0}^{\infty} \sum_{\epsilon_{n}}(-1)^{n} \frac{\lambda v_{F} p_{-}}{\left|\epsilon_{n}\right|} L_{n-1}^{1}\left(\frac{2 p_{\perp}^{2}}{\left|e B_{\lambda}\right|}\right) \\
& \times\left\{\frac{\theta\left(\epsilon_{n}\right)}{1+e^{\beta\left(\left|\epsilon_{n}\right|-\mu_{\lambda}\right)}}+\frac{\theta\left(-\epsilon_{n}\right)}{1+e^{\beta\left(\left|\epsilon_{n}\right|+\mu_{\lambda}\right)}}-\frac{1}{2}\right\} .
\end{aligned}
$$

Here $p_{\perp}^{2}=p_{1}^{2}+p_{2}^{2}, p_{ \pm}=p_{1} \pm i s_{B} p_{2}$, and we omitted the arguments of $W_{n m}$. Then the Wigner function of form (3.6) can be easily determined using the following relations:

$$
w_{0} \equiv \frac{W_{11}+W_{22}}{2}, \quad w_{1} \equiv \lambda \frac{W_{12}+W_{21}}{2}, \quad w_{2} \equiv i \lambda s_{B} \frac{W_{12}-W_{21}}{2}, \quad w_{3} \equiv \lambda s_{B} \frac{W_{11}-W_{22}}{2} .
$$

\section{Weak magnetic field limit}

In this appendix, we derive the Wigner function in the limit of a weak magnetic field. The coefficients $W_{11}, W_{22}, W_{12}$, and $W_{21}$ in eqs. (B.10) through (B.12) are

$$
\begin{aligned}
& W_{11} \equiv A_{-}-A_{+}+\delta A, \\
& W_{22} \equiv B_{-}-B_{+}+\delta B, \\
& W_{12} \equiv C_{-}+C_{+}+\delta C, \\
& W_{21} \equiv W_{12}^{*},
\end{aligned}
$$

where

$$
\begin{aligned}
& A_{\mp}=-e^{-p_{\perp}^{2} /\left|e B_{\lambda}\right|} \sum_{n=0}^{\infty}(-1)^{n} L_{n-1}\left(\frac{2 p_{\perp}^{2}}{\left|e B_{\lambda}\right|}\right) \frac{\left(\left|\epsilon_{n}\right| \mp s_{B} \lambda v_{F} p_{3}\right)}{\left|\epsilon_{n}\right|} \frac{\theta\left( \pm \epsilon_{n}\right)}{1+e^{\beta\left(\left|\epsilon_{n}\right| \mp \mu_{\lambda}\right)}}, \\
& B_{\mp}=e^{-p_{\perp}^{2} /\left|e B_{\lambda}\right|} \sum_{n=0}^{\infty}(-1)^{n} L_{n}\left(\frac{2 p_{\perp}^{2}}{\left|e B_{\lambda}\right|}\right) \frac{\left(\left|\epsilon_{n}\right| \pm s_{B} \lambda v_{F} p_{3}\right)}{\left|\epsilon_{n}\right|} \frac{\theta\left( \pm \epsilon_{n}\right)}{1+e^{\beta\left(\left|\epsilon_{n}\right| \mp \mu_{\lambda}\right)}} \\
& C_{\mp}=2 e^{-p_{\perp}^{2} /\left|e B_{\lambda}\right|} \sum_{n=0}^{\infty}(-1)^{n} L_{n-1}^{1}\left(\frac{2 p_{\perp}^{2}}{\left|e B_{\lambda}\right|}\right) \frac{\lambda v_{F} p_{-}}{\left|\epsilon_{n}\right|} \frac{\theta\left( \pm \epsilon_{n}\right)}{1+e^{\beta\left(\left|\epsilon_{n}\right| \mp \mu_{\lambda}\right)}}
\end{aligned}
$$

and

$$
\begin{aligned}
& \delta A=-e^{-p_{\perp}^{2} /\left|e B_{\lambda}\right|} \sum_{n=0}^{\infty}(-1)^{n} L_{n-1}\left(\frac{2 p_{\perp}^{2}}{\left|e B_{\lambda}\right|}\right) \frac{s_{B} \lambda v_{F} p_{3}}{\left|\epsilon_{n}\right|}, \\
& \delta B=-e^{-p_{\perp}^{2} /\left|e B_{\lambda}\right|} \sum_{n=0}^{\infty}(-1)^{n} L_{n}\left(\frac{2 p_{\perp}^{2}}{\left|e B_{\lambda}\right|}\right) \frac{s_{B} \lambda v_{F} p_{3}}{\left|\epsilon_{n}\right|}, \\
& \delta C=-2 e^{-p_{\perp}^{2} /\left|e B_{\lambda}\right|} \sum_{n=0}^{\infty}(-1)^{n} \frac{\lambda v_{F} p_{-}}{\left|\epsilon_{n}\right|} L_{n-1}^{1}\left(\frac{2 p_{\perp}^{2}}{\left|e B_{\lambda}\right|}\right) .
\end{aligned}
$$

In order to sum over all Landau levels, we employ the following tricks:

$$
\begin{aligned}
\frac{\left|\epsilon_{n}\right| \mp s_{B} \lambda v_{F} p_{3}}{\left|\epsilon_{n}\right|} \frac{\theta\left( \pm \epsilon_{n}\right)}{1+e^{\beta\left(\left|\epsilon_{n}\right| \mp \mu_{\lambda}\right)}} & =\sum_{m=0}^{\infty}\left(2 n\left|e B_{\lambda}\right|\right)^{m} \frac{G_{1}^{(m)}\left(p_{3}^{2}\right)}{m !} \\
& =\lim _{s_{1} \rightarrow-0} \sum_{m=0}^{\infty}\left(\frac{d^{m}}{d s_{1}^{m}} e^{2 s_{1} n\left|e B_{\lambda}\right|}\right) \frac{G_{1}^{(m)}\left(p_{3}^{2}\right)}{m !}
\end{aligned}
$$




$$
\begin{aligned}
& \frac{\left|\epsilon_{n}\right| \pm s_{B} \lambda v_{F} p_{3}}{\left|\epsilon_{n}\right|} \frac{\theta\left( \pm \epsilon_{n}\right)}{1+e^{\beta\left(\left|\epsilon_{n}\right| \mp \mu_{\lambda}\right)}}=\sum_{m=0}^{\infty}\left(2 n\left|e B_{\lambda}\right|\right)^{m} \frac{G_{2}^{(m)}\left(p_{3}^{2}\right)}{m !} \\
& =\lim _{s_{1} \rightarrow-0} \sum_{m=0}^{\infty}\left(\frac{d^{m}}{d s_{1}^{m}} e^{2 s_{1} n\left|e B_{\lambda}\right|}\right) \frac{G_{2}^{(m)}\left(p_{3}^{2}\right)}{m !}, \\
& \frac{1}{\left|\epsilon_{n}\right|} \frac{\theta\left( \pm \epsilon_{n}\right)}{1+e^{\beta\left(\left|\epsilon_{n}\right| \mp \mu_{\lambda}\right)}}=\sum_{m=0}^{\infty}\left(2 n\left|e B_{\lambda}\right|\right)^{m} \frac{G_{3}^{(m)}\left(p_{3}^{2}\right)}{m !} \\
& =\lim _{s_{1} \rightarrow-0} \sum_{m=0}^{\infty}\left(\frac{d^{m}}{d s_{1}^{m}} e^{2 s_{1} n\left|e B_{\lambda}\right|}\right) \frac{G_{3}^{(m)}\left(p_{3}^{2}\right)}{m !}, \\
& \frac{1}{\left|\epsilon_{n}\right|}=\sum_{m=0}^{\infty}\left(2 n\left|e B_{\lambda}\right|\right)^{m} \frac{G_{4}^{(m)}\left(p_{3}^{2}\right)}{m !}=\lim _{s_{1} \rightarrow-0} \sum_{m=0}^{\infty}\left(\frac{d^{m}}{d s_{1}^{m}} e^{2 s_{1} n\left|e B_{\lambda}\right|}\right) \frac{G_{4}^{(m)}\left(p_{3}^{2}\right)}{m !}
\end{aligned}
$$

where $G_{i}^{(m)}$ denotes the m-th derivative with respect to its argument and the following shorthand notations are used:

$$
\begin{aligned}
& G_{1}\left(p_{3}^{2}+2 n\left|e B_{\lambda}\right|\right)=\frac{\left|\epsilon_{n}\right| \mp s_{B} \lambda v_{F} p_{3}}{\left|\epsilon_{n}\right|} \frac{\theta\left( \pm \epsilon_{n}\right)}{1+e^{\beta\left(\left|\epsilon_{n}\right| \mp \mu_{\lambda}\right)}}, \\
& G_{2}\left(p_{3}^{2}+2 n\left|e B_{\lambda}\right|\right)=\frac{\left|\epsilon_{n}\right| \pm s_{B} \lambda v_{F} p_{3}}{\left|\epsilon_{n}\right|} \frac{\theta\left( \pm \epsilon_{n}\right)}{1+e^{\beta\left(\left|\epsilon_{n}\right| \mp \mu_{\lambda}\right)}}, \\
& G_{3}\left(p_{3}^{2}+2 n\left|e B_{\lambda}\right|\right)=\frac{1}{\left|\epsilon_{n}\right|} \frac{\theta\left( \pm \epsilon_{n}\right)}{1+e^{\beta\left(\left|\epsilon_{n}\right| \mp \mu_{\lambda}\right)}}, \\
& G_{4}\left(p_{3}^{2}+2 n\left|e B_{\lambda}\right|\right)=\frac{1}{\left|\epsilon_{n}\right|} .
\end{aligned}
$$

Performing the summation over Landau levels by using formula 7.414 .8 in ref. [75], we obtain

$$
\begin{aligned}
& A_{\mp}=e^{-p_{\perp}^{2} /\left|e B_{\lambda}\right|} \lim _{s_{1} \rightarrow-0} \sum_{m=0}^{\infty} \frac{d^{m}}{d s_{1}^{m}}\left[e^{2 s_{1}\left|e B_{\lambda}\right|} \frac{\exp \left(\frac{p_{\perp}^{2}}{\left|e B_{\lambda}\right|\left(1+e^{-2 s_{1}\left|e B_{\lambda}\right|}\right)}\right)}{1+e^{2 s_{1}\left|e B_{\lambda}\right|}} \frac{G_{1}^{(m)}\left(p_{3}^{2}\right)}{m !}\right] \\
& \simeq \frac{1}{2} \sum_{m=0}^{\infty} \frac{\left(p_{\perp}^{2}\right)^{m}}{m !} G_{1}^{(m)}\left(p_{3}^{2}\right)+\frac{\left|e B_{\lambda}\right|}{2} \sum_{m=0}^{\infty} \frac{\left(p_{\perp}^{2}\right)^{m-1}}{(m-1) !} G_{1}^{(m)}\left(p_{3}^{2}\right)+O\left(\left|e B_{\lambda}\right|^{2}\right) \\
& \simeq \frac{1}{2} G_{1}\left(\epsilon_{\mathbf{p}}\right)+\frac{\left|e B_{\lambda}\right|}{2}\left(\frac{d}{d p_{\perp}^{2}} G_{1}\left(\epsilon_{\mathbf{p}}\right)\right)+O\left(\left|e B_{\lambda}\right|^{2}\right) \\
& B_{\mp}=e^{-p_{\perp}^{2} /\left|e B_{\lambda}\right|} \lim _{s_{1} \rightarrow-0} \sum_{m=0}^{\infty} \frac{d^{m}}{d s_{1}^{m}}\left[\frac{\exp \left(\frac{p_{\perp}^{2}}{\left|e B_{\lambda}\right|\left(1+e^{-2 s_{1}\left|e B_{\lambda}\right|}\right)}\right)}{1+e^{2 s_{1}\left|e B_{\lambda}\right|}} \frac{G_{2}^{(m)}\left(p_{3}^{2}\right)}{m !}\right] \\
& \simeq \frac{1}{2} \sum_{m=0}^{\infty} \frac{\left(p_{\perp}^{2}\right)^{m}}{m !} G_{2}^{(m)}\left(p_{3}^{2}\right)-\frac{\left|e B_{\lambda}\right|}{2} \sum_{m=0}^{\infty} \frac{\left(p_{\perp}^{2}\right)^{m-1}}{(m-1) !} G_{2}^{(m)}\left(p_{3}^{2}\right)+O\left(\left|e B_{\lambda}\right|^{2}\right) \\
& \simeq \frac{1}{2} G_{2}\left(\epsilon_{\mathbf{p}}\right)-\frac{\left|e B_{\lambda}\right|}{2}\left(\frac{d}{d p_{\perp}^{2}} G_{2}\left(\epsilon_{\mathbf{p}}\right)\right)+O\left(\left|e B_{\lambda}\right|^{2}\right)
\end{aligned}
$$




$$
\begin{aligned}
C_{\mp} & =-2 \lambda v_{F} p_{-} e^{-p_{\perp}^{2} /\left|e B_{\lambda}\right|} \lim _{s_{1} \rightarrow-0} \sum_{m=0}^{\infty} \frac{d^{m}}{d s_{1}^{m}}\left[e^{2 s_{1}\left|e B_{\lambda}\right|} \frac{\exp \left(\frac{p_{\perp}^{2}}{\left|e B_{\lambda}\right|\left(1+e^{-2 s_{1}\left|e B_{\lambda}\right|}\right)}\right)}{\left(1+e^{2 s_{1}\left|e B_{\lambda}\right|}\right)^{2}} \frac{G_{3}^{(m)}\left(p_{3}^{2}\right)}{m !}\right] \\
& \simeq-\frac{\lambda v_{F} p_{-}}{2} \sum_{m=0}^{\infty} \frac{\left(p_{\perp}^{2}\right)^{m}}{m !} G_{3}^{(m)}\left(p_{3}^{2}\right)+O\left(\left|e B_{\lambda}\right|^{2}\right) \simeq-\frac{\lambda v_{F} p_{-}}{2} G_{3}\left(\epsilon_{\mathbf{p}}\right)+O\left(\left|e B_{\lambda}\right|^{2}\right), \quad \text { (C.21) }
\end{aligned}
$$

where $\epsilon_{\mathbf{p}} \equiv v_{F}|\mathbf{p}|$. The coefficients $\delta A, \delta B$, and $\delta C$ read

$$
\begin{aligned}
& \delta A=s_{B} \lambda v_{F} p_{3} e^{-p_{\perp}^{2} /\left|e B_{\lambda}\right|} \lim _{s_{1} \rightarrow-0} \sum_{m=0}^{\infty} \frac{d^{m}}{d s_{1}^{m}}\left[e^{2 s_{1}\left|e B_{\lambda}\right|} \frac{\exp \left(\frac{p_{\perp}^{2}}{\left|e B_{\lambda}\right|\left(1+e^{-2 s_{1}\left|e B_{\lambda}\right|}\right)}\right)}{1+e^{2 s_{1}\left|e B_{\lambda}\right|}} \frac{G_{4}^{(m)}\left(p_{3}^{2}\right)}{m !}\right] \\
& \simeq \frac{s_{B} \lambda v_{F} p_{3}}{2} \sum_{m=0}^{\infty} \frac{\left(p_{\perp}^{2}\right)^{m}}{m !} G_{4}^{(m)}\left(p_{3}^{2}\right)+s_{B} \lambda v_{F} p_{3} \frac{\left|e B_{\lambda}\right|}{2} \sum_{m=0}^{\infty} \frac{\left(p_{\perp}^{2}\right)^{m-1}}{(m-1) !} G_{4}^{(m)}\left(p_{3}^{2}\right)+O\left(\left|e B_{\lambda}\right|^{2}\right) \\
& \simeq \frac{s_{B} \lambda v_{F} p_{3}}{2} G_{4}\left(\epsilon_{\mathbf{p}}\right)+s_{B} \lambda p_{3} \frac{\left|e B_{\lambda}\right|}{2}\left(\frac{d}{d p_{\perp}^{2}} G_{4}\left(\epsilon_{\mathbf{p}}\right)\right)+O\left(\left|e B_{\lambda}\right|^{2}\right) \\
& =\frac{s_{B} \lambda v_{F} p_{3}}{2 \epsilon_{\mathbf{p}}}-s_{B} \lambda v_{F}^{3} p_{3} \frac{\left|e B_{\lambda}\right|}{4 \epsilon_{\mathbf{p}}^{3}}+O\left(\left|e B_{\lambda}\right|^{2}\right), \\
& \delta B=-s_{B} \lambda v_{F} p_{3} e^{-p_{\perp}^{2} /\left|e B_{\lambda}\right|} \lim _{s_{1} \rightarrow-0} \sum_{m=0}^{\infty} \frac{d^{m}}{d s_{1}^{m}}\left[\frac{\exp \left(\frac{p_{\perp}^{2}}{\left|e B_{\lambda}\right|\left(1+e^{-2 s_{1}\left|e B_{\lambda}\right|}\right)}\right)}{1+e^{2 s_{1}\left|e B_{\lambda}\right|}} \frac{G_{4}^{(m)}\left(p_{3}^{2}\right)}{m !}\right] \\
& \simeq \frac{-s_{B} \lambda v_{F} p_{3}}{2} \sum_{m=0}^{\infty} \frac{\left(p_{\perp}^{2}\right)^{m}}{m !} G_{4}^{(m)}\left(p_{3}^{2}\right)+s_{B} \lambda v_{F} p_{3} \frac{\left|e B_{\lambda}\right|}{2} \sum_{m=0}^{\infty} \frac{\left(p_{\perp}^{2}\right)^{m-1}}{(m-1) !} G_{4}^{(m)}\left(p_{3}^{2}\right)+O\left(\left|e B_{\lambda}\right|^{2}\right) \\
& \simeq-\frac{s_{B} \lambda v_{F} p_{3}}{2} G_{4}\left(\epsilon_{\mathbf{p}}\right)+s_{B} \lambda v_{F} p_{3} \frac{\left|e B_{\lambda}\right|}{2}\left(\frac{d}{d p_{\perp}^{2}} G_{4}\left(\epsilon_{\mathbf{p}}\right)\right)+O\left(\left|e B_{\lambda}\right|^{2}\right) \\
& =-\frac{s_{B} \lambda v_{F} p_{3}}{2 \epsilon_{\mathbf{p}}}-s_{B} \lambda v_{F}^{3} p_{3} \frac{\left|e B_{\lambda}\right|}{4 \epsilon_{\mathbf{p}}^{3}}+O\left(\left|e B_{\lambda}\right|^{2}\right), \\
& \delta C=-2 \lambda v_{F} p_{-} e^{-p_{\perp}^{2} /\left|e B_{\lambda}\right|} \lim _{s_{1} \rightarrow-0}(-1) \sum_{m=0}^{\infty} \frac{d^{m}}{d s_{1}^{m}}\left[e^{2 s_{1}\left|e B_{\lambda}\right|} \frac{\exp \left(\frac{p_{\perp}^{2}}{\left|e B_{\lambda}\right|\left(1+e^{-2 s_{1}\left|e B_{\lambda}\right|}\right)}\right)}{\left(1+e^{2 s_{1}\left|e B_{\lambda}\right|}\right)^{2}} \frac{G_{4}^{(m)}\left(p_{3}^{2}\right)}{m !}\right]
\end{aligned}
$$$$
\simeq \frac{\lambda v_{F} p_{-}}{2} \sum_{m=0}^{\infty} \frac{\left(p_{\perp}^{2}\right)^{m}}{m !} G_{3}^{(m)}\left(p_{3}^{2}\right)+O\left(\left|e B_{\lambda}\right|^{2}\right)=\frac{\lambda v_{F} p_{-}}{2} G_{3}\left(\epsilon_{\mathbf{p}}\right)+O\left(\left|e B_{\lambda}\right|^{2}\right)
$$$$
=\frac{\lambda v_{F} p_{-}}{2 \epsilon_{\mathbf{p}}}+O\left(\left|e B_{\lambda}\right|^{2}\right) \text {. }
$$

Combining the above results together and using eq. (B.13), we obtain eqs. (3.15a) and $(3.15 b)$ in the main text.

\section{Useful formulas}

In this appendix, we present some key formulas used in the calculation of the electric and heat current densities, defined by eqs. (6.4a) and (6.4b) in the main text. 
Let us start by presenting the result for the following table integral:

$$
\int_{0}^{\infty} d p_{3} p_{3}^{n} \frac{1}{1+e^{\left(v_{F} p_{3} \mp \mu_{\lambda}\right) / T}}=-\frac{T^{n+1} \Gamma(n+1)}{v_{F}^{n+1}} \operatorname{Li}_{n+1}\left(-e^{ \pm \mu_{\lambda} / T}\right), \quad n \geq 0,
$$

where $\operatorname{Li}_{n}(x)$ is the polylogarithm function (see formula 1.1.14 in ref. [76]). [Note that in the given reference $\operatorname{Li}_{n}(x) \equiv \mathrm{F}(x, n)$.] The polylogarithm function at $n=0,1$ can be rewritten as follows:

$$
\begin{aligned}
& \operatorname{Li}_{0}\left(-e^{x}\right)=-\frac{1}{1+e^{-x}}, \\
& \operatorname{Li}_{1}\left(-e^{x}\right)=-\ln \left(1+e^{x}\right) .
\end{aligned}
$$

The following identities for the polylogarithm functions are useful when taking into account the antiparticles contributions:

$$
\begin{aligned}
\ln \left(1+e^{x}\right)-\ln \left(1+e^{-x}\right) & =x, \\
\operatorname{Li}_{2}\left(-e^{x}\right)+\operatorname{Li}_{2}\left(-e^{-x}\right) & =-\frac{x^{2}}{2}-\frac{\pi^{2}}{6} .
\end{aligned}
$$

By making use of the table integral in eq. (D.1), it is straightforward to check the following results for the four types of integrations encountered in the calculation of the electric and heat current densities:

$$
\begin{aligned}
I_{1} & =\int \frac{d^{3} \mathbf{p}}{(2 \pi)^{3}} e^{-p_{\perp}^{2} /\left|e B_{0, \lambda}\right|} \tilde{f}_{L L L}\left(p_{3}\right)=\frac{\left|e B_{0, \lambda}\right|}{2(2 \pi)^{2}} \frac{\mu_{\lambda}}{v_{F}} \\
I_{2} & =\int \frac{d^{3} \mathbf{p}}{(2 \pi)^{3}} e^{-p_{\perp}^{2} /\left|e B_{0, \lambda}\right|} s_{B} \lambda v_{F} p_{3} \tilde{f}_{L L L}\left(p_{3}\right)=\frac{\left|e B_{0, \lambda}\right|}{4 v_{F}(2 \pi)^{2}}\left(\mu_{\lambda}^{2}+\frac{\pi^{2} T^{2}}{3}-v_{F}^{2} \Lambda^{2}\right), \\
I_{3} & =\int \frac{d^{3} \mathbf{p}}{(2 \pi)^{3}} e^{-p_{\perp}^{2} /\left|e B_{0, \lambda}\right|} \partial_{p_{3}} \tilde{f}_{L L L}\left(p_{3}\right)=-\frac{e B_{0, \lambda}}{2(2 \pi)^{2}} \lambda, \\
I_{4} & =\int \frac{d^{3} \mathbf{p}}{(2 \pi)^{3}} e^{-p_{\perp}^{2} /\left|e B_{0, \lambda}\right|} s_{B} \lambda v_{F} p_{3} \partial_{p_{3}} \tilde{f}_{L L L}\left(p_{3}\right)=-\frac{e B_{0, \lambda}}{2(2 \pi)^{2}} \lambda \mu_{\lambda},
\end{aligned}
$$

where $\tilde{f}_{L L L}\left(p_{3}\right)$ is the Wigner function in the LLL approximation defined in eq. (4.10). It should be noted that the last term in the parentheses on the right-hand side of eq. (D.7) contains a quadratic divergency that stems from $-\lambda s_{B} \operatorname{sgn}\left(p_{3}\right) / 2$ term in the function $\tilde{f}_{L L L}\left(p_{3}\right)$.

Open Access. This article is distributed under the terms of the Creative Commons Attribution License (CC-BY 4.0), which permits any use, distribution and reproduction in any medium, provided the original author(s) and source are credited.

\section{References}

[1] E.M. Lifshitz and L.P. Pitaevskii, Physical Kinetics, Pergamon Press, New York, U.S.A. (1981).

[2] R.L. Liboff, Kinetic Theory: Classic, Quantum, and Relativistic Descriptions, Springer-Verlag, New York, U.S.A. (2003). 
[3] J.P. Vallee, Magnetic fields in the galactic Universe, as observed in supershells, galaxies, intergalactic and cosmic realms, New Astron. Rev. 55 (2011) 91 [INSPIRE].

[4] R. Durrer and A. Neronov, Cosmological Magnetic Fields: Their Generation, Evolution and Observation, Astron. Astrophys. Rev. 21 (2013) 62 [arXiv:1303.7121] [INSPIRE].

[5] D.E. Kharzeev, L.D. McLerran and H.J. Warringa, The effects of topological charge change in heavy ion collisions: 'Event by event P and CP-violation', Nucl. Phys. A 803 (2008) 227 [arXiv:0711.0950] [INSPIRE].

[6] D.E. Kharzeev, J. Liao, S.A. Voloshin and G. Wang, Chiral magnetic and vortical effects in high-energy nuclear collisions - A status report, Prog. Part. Nucl. Phys. 88 (2016) 1 [arXiv: 1511.04050] [INSPIRE].

[7] C. Kouveliotou et al., Discovery of a magnetar associated with the soft gamma repeater SGR 1900+14, Astrophys. J. 510 (1999) L115 [astro-ph/9809140] [INSPIRE].

[8] S. Borisenko, Q. Gibson, D. Evtushinsky, V. Zabolotnyy, B. Buchner and R.J. Cava, Experimental Realization of a Three-Dimensional Dirac Semimetal, Phys. Rev. Lett. 113 (2014) 027603 [arXiv: 1309.7978].

[9] M. Neupane et al., Observation of a topological 3D Dirac semimetal phase in high-mobility $\mathrm{Cd}_{3} A s_{2}$, . Nature Commun. 5 (2014) 3786 [arXiv:1309.7892].

[10] Z.K. Liu et al., Discovery of a Three-Dimensional Topological Dirac Semimetal, $\mathrm{Na}_{3} \mathrm{Bi}$, Science 343 (2014) 864 [arXiv:1310.0391].

[11] J. Xiong et al., Evidence for the chiral anomaly in the Dirac semimetal $N a_{3} B i$, Science 350 (2015) 413.

[12] C.-Z. Li, L.-X. Wang, H. Liu, J. Wang, Z.-M. Liao and D.-P. Yu, Giant negative magnetoresistance induced by the chiral anomaly in individual $\mathrm{Cd}_{3} A s_{2}$ nanowires, Nature Commun. 6 (2015) 10137 [arXiv: 1504.07398].

[13] H. Li et al., Negative magnetoresistance in Dirac semimetal $C_{3} A s_{2}$, Nat. Commun. 7 (2016) 10301.

[14] Q. Li et al., Observation of the chiral magnetic effect in ZrTe5, Nature Phys. 12 (2016) 550 [arXiv: 1412.6543] [INSPIRE].

[15] H.M. Weng, C. Fang, Z. Fang, B.A. Bernevig and X. Dai, Weyl semimetal phase in noncentrosymmetric transition-metal monophosphides, Phys. Rev. X 5 (2015) 011029.

[16] B.Q. Lv et al., Experimental discovery of Weyl semimetal TaAs, Phys. Rev. X 5 (2015) 031013 [arXiv: 1502.04684] [INSPIRE].

[17] X. Huang et al., Observation of the Chiral-Anomaly-Induced Negative Magnetoresistance in $3 D$ Weyl Semimetal TaAs, Phys. Rev. X 5 (2015) 031023.

[18] S.-Y. Xu et al., Discovery of a Weyl fermion semimetal and topological Fermi arcs, Science 349 (2015) 613 [arXiv:1502.03807].

[19] S.-M. Huang et al., A Weyl fermion semimetal with surface Fermi arcs in the transition metal monopnictide TaAs class, Nat. Commun. 6 (2015) 7373.

[20] C.-L. Zhang et al., Signatures of the Adler-Bell-Jackiw chiral anomaly in a Weyl fermion semimetal, Nat. Commun. 7 (2016) 10735.

[21] S. Borisenko et al., Time-Reversal Symmetry Breaking Type-II Weyl State in YbMnBi $i_{2}$, arXiv: 1507.04847. 
[22] I. Belopolski et al., Unoccupied electronic structure and signatures of topological Fermi arcs in the Weyl semimetal candidate $M o_{x} W_{1-x} T e_{2}$, arXiv:1512.09099.

[23] S.L. Adler, Axial vector vertex in spinor electrodynamics, Phys. Rev. 177 (1969) 2426 [INSPIRE].

[24] J.S. Bell and R. Jackiw, A PCAC puzzle: $\pi_{0} \rightarrow \gamma \gamma$ in the $\sigma$-model, Nuovo Cim. A 60 (1969) 47 [INSPIRE].

[25] D.T. Son and N. Yamamoto, Berry Curvature, Triangle Anomalies and the Chiral Magnetic Effect in Fermi Liquids, Phys. Rev. Lett. 109 (2012) 181602 [arXiv:1203.2697] [InSPIRE].

[26] D.T. Son and N. Yamamoto, Kinetic theory with Berry curvature from quantum field theories, Phys. Rev. D 87 (2013) 085016 [arXiv:1210.8158] [INSPIRE].

[27] M.A. Stephanov and Y. Yin, Chiral Kinetic Theory, Phys. Rev. Lett. 109 (2012) 162001 [arXiv: 1207.0747] [INSPIRE].

[28] N. Nagaosa, J. Sinova, Sh. Onoda, A.H. MacDonald and N.P. Ong, Anomalous Hall effect, Rev. Mod. Phys. 82 (2010) 1539 [arXiv:0904.4154].

[29] M.V. Berry, Quantal phase factors accompanying adiabatic changes, Proc. Roy. Soc. Lond. A 392 (1984) 45 [INSPIRE].

[30] D. Xiao, M.-C. Chang and Q. Niu, Berry Phase Effects on Electronic Properties, Rev. Mod. Phys. 82 (2010) 1959 [arXiv:0907.2021] [InSPIRE].

[31] K. Fukushima, D.E. Kharzeev and H.J. Warringa, The Chiral Magnetic Effect, Phys. Rev. D 78 (2008) 074033 [arXiv:0808.3382] [INSPIRE].

[32] Y. Gao, S.A. Yang and Q. Niu, Field Induced Positional Shift of Bloch Electrons and Its Dynamical Implications, Phys. Rev. Lett. 112 (2014) 166601 [arXiv:1402.2538].

[33] Y. Gao, S.A. Yang and Q. Niu, Geometrical effects in orbital magnetic susceptibility, Phys. Rev. B 91 (2015) 214405 [arXiv: 1411.0324].

[34] E.V. Gorbar, V.A. Miransky, I.A. Shovkovy and P.O. Sukhachov, Second-order chiral kinetic theory: Chiral magnetic and pseudomagnetic waves, Phys. Rev. B 95 (2017) 205141 [arXiv: 1702.02950] [INSPIRE].

[35] E.P. Wigner, On the quantum correction for thermodynamic equilibrium, Phys. Rev. 40 (1932) 749 [INSPIRE].

[36] H.T. Elze, M. Gyulassy and D. Vasak, Transport Equations for the QCD Quark Wigner Operator, Nucl. Phys. B 276 (1986) 706 [InSPIRE].

[37] D. Vasak, M. Gyulassy and H.T. Elze, Quantum Transport Theory for Abelian Plasmas, Annals Phys. 173 (1987) 462 [inSPIRE].

[38] H.-T. Elze and U.W. Heinz, Quark-Gluon Transport Theory, Phys. Rept. 183 (1989) 81 [INSPIRE].

[39] K.S. Zachos, D.B. Fairlie and Th.L. Curtright, Quantum Mechanics in Phase Space, World Scientific, New Jersey, U.S.A. (2005).

[40] A. Polkovnikov, Phase space representation of quantum dynamics, Annals Phys. 325 (2010) 1790 [arXiv: 0905.3384] [INSPIRE].

[41] E.A. Calzetta and B.-L.B. Hu, Nonequilibrium Quantum Field Theory, Cambridge University Press, (2008). 
[42] J.-W. Chen, S. Pu, Q. Wang and X.-N. Wang, Berry Curvature and Four-Dimensional Monopoles in the Relativistic Chiral Kinetic Equation, Phys. Rev. Lett. 110 (2013) 262301 [arXiv:1210.8312] [INSPIRE].

[43] I. Bialynicki-Birula, P. Gornicki and J. Rafelski, Phase space structure of the Dirac vacuum, Phys. Rev. D 44 (1991) 1825 [INSPIRE].

[44] F. Hebenstreit, R. Alkofer and H. Gies, Schwinger pair production in space and time-dependent electric fields: Relating the Wigner formalism to quantum kinetic theory, Phys. Rev. D 82 (2010) 105026 [arXiv:1007.1099] [InSPIRE].

[45] D. Xiao, Y. Yao, Z. Fang and Q. Niu, Berry-Phase Effect in Anomalous Thermoelectric Transport, Phys. Rev. Lett. 97 (2006) 026603 [cond-mat/0604561].

[46] T. Qin, Q. Niu and J. Shi, Energy Magnetization and the Thermal Hall Effect, Phys. Rev. Lett. 107 (2011) 236601 [arXiv:1108.3879].

[47] R. Lundgren, P. Laurell and G.A. Fiete, Thermoelectric properties of Weyl and Dirac semimetals, Phys. Rev. B 90 (2014) 165115 [arXiv:1407.1435] [INSPIRE].

[48] G. Sharma, P. Goswami and S. Tewari, Nernst and magnetothermal conductivity in a lattice model of Weyl fermions, Phys. Rev. B 93 (2016) 035116 [arXiv:1507.05606] [INSPIRE].

[49] G. Sharma, C. Moore and S. Tewari, Nernst effect in topological Dirac semimetals, arXiv: 1605.00299 [INSPIRE].

[50] C.J. Tabert, J.P. Carbotte and E.J. Nicol, Optical and Transport Properties in 3D Dirac and Weyl Semimetals, Phys. Rev. B 94 (2016) 039901 [arXiv:1603.00866].

[51] Q. Chen and G.A. Fiete, Thermoelectric transport in double-Weyl semimetals, Phys. Rev. B 93 (2016) 155125 [arXiv: 1601.03087].

[52] J. Zhou, H. Jiang, Q. Niu and J. Shi, Topological Invariants of Metals and Related Physical Effects, Chin. Phys. Lett. 30 (2013) 027101 [arXiv:1211.0772] [InSPIRE].

[53] M.A. Zubkov, Emergent gravity and chiral anomaly in Dirac semimetals in the presence of dislocations, Annals Phys. 360 (2015) 655 [arXiv: 1501.04998] [INSPIRE].

[54] A. Cortijo, Y. Ferreiros, K. Landsteiner and M.A.H. Vozmediano, Elastic Gauge Fields in Weyl Semimetals, Phys. Rev. Lett. 115 (2015) 177202 [arXiv:1603.02674] [INSPIRE].

[55] A. Cortijo, D. Kharzeev, K. Landsteiner and M.A.H. Vozmediano, Strain induced Chiral Magnetic Effect in Weyl semimetals, Phys. Rev. B 94 (2016) 241405 [arXiv:1607.03491] [INSPIRE].

[56] A.G. Grushin, J.W.F. Venderbos, A. Vishwanath and R. Ilan, Inhomogeneous Weyl and Dirac semimetals: Transport in axial magnetic fields and Fermi arc surface states from pseudo Landau levels, Phys. Rev. X 6 (2016) 041046 [arXiv: 1607.04268].

[57] D.I. Pikulin, A. Chen and M. Franz, Chiral anomaly from strain-induced gauge fields in Dirac and Weyl semimetals, Phys. Rev. X 6 (2016) 041021 [arXiv:1607.01810] [InSPIRE].

[58] T. Liu, D.I. Pikulin and M. Franz, Quantum oscillations without magnetic field, Phys. Rev. B 95 (2017) 041201 [arXiv: 1608.04678].

[59] S.R. de Groot and G.L. Suttorp, Foundation of Electrodynamics, North-Holland, Amsterdam, The Netherlands (1972).

[60] S.R. de Groot, W.A. van Leeuwen, and S.G. van Weert, Relativistic Kinetic Theory, North-Holland, Amsterdam, The Netherlands (1980). 
[61] C. Best, P. Gornicki and W. Greiner, The phase space structure of the Klein-Gordon field, Annals Phys. 225 (1993) 169 [hep-ph/9301275] [INSPIRE].

[62] G.R. Shin and J. Rafelski, Relativistic transport equations for electromagnetic, scalar and pseudoscalar potentials, Annals Phys. 243 (1995) 65 [INSPIRE].

[63] J.S. Schwinger, On the Green's functions of quantized fields. I., Proceed. Nat. Acad. Sciences 37 (1951) 452.

[64] A. Vilenkin, Cancellation of equilibrium parity violating currents, Phys. Rev. D 22 (1980) 3067 [INSPIRE].

[65] M.A. Metlitski and A.R. Zhitnitsky, Anomalous axion interactions and topological currents in dense matter, Phys. Rev. D 72 (2005) 045011 [hep-ph/0505072] [INSPIRE].

[66] J.-H. Gao, Z.-T. Liang, S. Pu, Q. Wang and X.-N. Wang, Chiral Anomaly and Local Polarization Effect from Quantum Kinetic Approach, Phys. Rev. Lett. 109 (2012) 232301 [arXiv: 1203.0725] [INSPIRE].

[67] J.-h. Gao, S. Pu and Q. Wang, Covariant chiral kinetic equation in the Wigner function approach, Phys. Rev. D 96 (2017) 016002 [arXiv:1704.00244] [INSPIRE].

[68] D.E. Kharzeev and H.-U. Yee, Chiral Magnetic Wave, Phys. Rev. D 83 (2011) 085007 [arXiv: 1012.6026] [INSPIRE].

[69] E.V. Gorbar, V.A. Miransky, I.A. Shovkovy and P.O. Sukhachov, Consistent Chiral Kinetic Theory in Weyl Materials: Chiral Magnetic Plasmons, Phys. Rev. Lett. 118 (2017) 127601 [arXiv: 1610.07625] [INSPIRE].

[70] E.V. Gorbar, V.A. Miransky, I.A. Shovkovy and P.O. Sukhachov, Chiral magnetic plasmons in anomalous relativistic matter, Phys. Rev. B 95 (2017) 115202 [arXiv:1611.05470] [INSPIRE].

[71] K. Fukushima, K. Hattori, H.-U. Yee and Y. Yin, Heavy Quark Diffusion in Strong Magnetic Fields at Weak Coupling and Implications for Elliptic Flow, Phys. Rev. D 93 (2016) 074028 [arXiv: 1512.03689] [INSPIRE].

[72] V.P. Gusynin, V.A. Miransky and I.A. Shovkovy, Dynamical chiral symmetry breaking in QED in a magnetic field: Toward exact results, Phys. Rev. Lett. 83 (1999) 1291 [hep-th/9811079] [INSPIRE].

[73] X.-l. Sheng, D.H. Rischke, D. Vasak and Q. Wang, Wigner functions of massive fermions in strong magnetic fields, arXiv:1707.01388 [INSPIRE].

[74] H. Bateman, A. Erdelyi, Higher Transcendental Functions, Vol. 2, McGraw-Hill Book Company, New York, U.S.A. (1953).

[75] I.S. Gradshtein and I.M. Ryzhik, Tables of Integrals, Series, and Products, Academic Press, Orlando, U.S.A. (1980).

[76] A. Erdelyi, W. Magnus, F. Oberhettinger and F.G. Tricomi, Higher Transcendental Functions, Vol. 1, Krieger, New York, U.S.A. (1981). 